\title{
A structure of human Scap bound to Insig-2 suggests how their interaction is regulated by sterols
}

\author{
Renhong Yan ${ }^{1,2 *}$, Pingping Cao ${ }^{3 *}$, Wenqi Song ${ }^{3 *}$, Hongwu Qian ${ }^{4}$, Ximing Du ${ }^{5}$ Hudson W. Coates ${ }^{5}$, Xin Zhao $^{3}$, \\ Yaning Li ${ }^{3}$, Shuai Gao ${ }^{4}$, Xin Gong ${ }^{6}$, Ximing Liu ${ }^{7}$, Jianhua Sui ${ }^{7,8}$, Jianlin Lei ${ }^{9}$, Hongyuan Yang ${ }^{5}$, Andrew J. \\ Brown $^{5}$, Qiang Zhou ${ }^{1,2}$, Chuangye Yan ${ }^{3}+$, Nieng Yan ${ }^{4} \uparrow$ \\ ${ }^{1}$ Westlake Laboratory of Life Sciences and Biomedicine, Key Laboratory of Structural Biology of Zhejiang Province, School of Life Sciences, Westlake University, Hangzhou \\ 310024, Zhejiang Province, China. Institute of Biology, Westlake Institute for Advanced Study, Hangzhou 310024, Zhejiang Province, China. ${ }^{3}$ State Key Laboratory of \\ Membrane Biology, Beijing Advanced Innovation Center for Structural Biology, Tsinghua-Peking Joint Center for Life Sciences, School of Life Sciences, Tsinghua University, \\ Beijing 100084, China. ${ }^{4}$ Department of Molecular Biology, Princeton University, Princeton, NJ 08544, USA. ${ }^{5}$ School of Biotechnology and Biomolecular Science, University \\ of New South Wales, Sydney, NSW 2052, Australia. ${ }^{6}$ Department of Biology, Southern University of Science and Technology, Shenzhen, Guangdong 518055, China. \\ ${ }^{7}$ National Institute of Biological Sciences (NIBS), Beijing 102206, China. ${ }^{8}$ Tsinghua Institute of Multidisciplinary Biomedical Research, Tsinghua University, Beijing 102206, \\ China. ${ }^{9}$ Technology Center for Protein Sciences, Ministry of Education Key Laboratory of Protein Sciences, School of Life Sciences, Tsinghua University, Beijing 100084, \\ China.
}

*These authors contributed equally to this work.

†Corresponding author. Email: nyan@princeton.edu (N.Y.); yancy2019@tsinghua.edu.cn (C.Y.)

The SREBP pathway controls cellular homeostasis of sterols. The key players in this pathway, Scap and Insig-1/2, are membrane-embedded sterol sensors. 25-hydroxycholesterol (25HC)-dependent association of Scap and Insigs acts as the master switch for the SREBP pathway. Here, we present cryo-EM analysis of the human Scap and Insig-2 complex in the presence of $25 \mathrm{HC}$, with the transmembrane (TM) domains determined at an average resolution of $3.7 \AA$. The sterol sensing domain (SSD) in Scap and all six TMs in Insig-2 were resolved. A 25HC molecule is sandwiched between the S4-S6 segments in Scap and TMs 3/4 in Insig-2 in the luminal leaflet of the membrane. Unwinding of the middle of the Scap-S4 segment is crucial for $25 \mathrm{HC}$ binding and Insig association.

The sterol regulatory element-binding protein (SREBP) pathway controls cellular homeostasis of sterols through a negative feedback mechanism (1, 2) (Fig. 1A). SREBPs (SREBP-1a, 1c, 2) are transcription factors in which a transmembrane hairpin connects the amino $(\mathrm{N})$ terminal transcription factor domain and the carboxy (C) terminal regulatory domain (3-5). The C-terminal domain of SREBP is constitutively bound to the C-terminal WD40 domain of the SREBP-cleavage activating protein (Scap) $(6,7)$. Preceding the WD40 domain, Scap comprises eight transmembrane (TM) segments, with segments S2-S6 constituting the sterolsensing domain (SSD) (8-10) (Fig. 1A).

The membrane-bound transcription factor SREBP-2 regulates cholesterol metabolism. When the cell is enriched with cholesterol, the SREBP-2/Scap complex is sequestered to the endoplasmic reticulum (ER) membrane through interaction between the Scap-SSD and Insig-1 or 2 (insulin-induced gene) (11-13). The association between Scap and Insig requires a cholesterol derivative like 25-hydroxycholesterol (25HC), or less favorably, cholesterol (14). Upon cholesterol depletion, the SREBP-2/Scap complex dissociates from Insig-1/2 and is transported by COPII-coated vesicles from the ER to the Golgi, where SREBP-2 undergoes two sequential cleavages.
The linker between the two TMs is first cut in the lumen by the site-1 protease (S1P), then TM1 is cleaved by the site- 2 protease (S2P), an integral membrane metalloprotease, within the lipid bilayer (15-19). The transcription factor domain of SREBP-2 is released and translocated into the nucleus to activate the expression of genes encoding proteins for cholesterol uptake, exemplified by the low-density lipoprotein (LDL) receptor and enzymes for cholesterol synthesis, such as the rate-limiting enzyme 3-hydroxy-3-methylglutarylcoenzyme A reductase (HMGCR) (1, 20-22).

HMGCR also contains a SSD that interacts with Insig-1/2 in a sterol-dependent manner (fig. S1A). Insig-1 bridges the interaction between an E3 ubiquitin ligase, GP78, and HMGCR for degradation of the latter $(2,23-25)$. Therefore, the interaction between Insig and the SSD in Scap and HMGCR is essential for the feedback inhibition to maintain cellular cholesterol homeostasis.

Although the pathway was discovered more than two decades ago, structural elucidation of the players has lagged behind the cellular and biochemical characterizations. Structural understanding of this pathway has mainly come from homologs in lower organisms, such as the crystal structures of an archaeal S2P MjS2P (26), a mycobacterial Insig 
MvINS (27), and the C-terminal domains of the SREBP and Scap homologs in S. pombe, Sre1 and Scp1 $(28,29)$. The cryoEM structures of several SSD-containing proteins (NiemannPick type C (NPC1), Patched 1 (Ptch1), NPC1L1, and Dispatched) have also been resolved (fig. S1A) (30-38). A putative sterol binding pocket is observed on the membranefacing surface of the SSD in Ptch1 and NPC1L1, enclosed by S2-S4 (the S2-S4 site) (32-34, 38).

Despite these advances, the $25 \mathrm{HC}$ binding site and the interface between the SSD and Insig remain enigmatic. In addition, Insig was shown to bind to $25 \mathrm{HC}$, but not cholesterol, whereas Scap can only bind to cholesterol through its luminal domain known as Loop 1 (39-41). Here we combine biochemistry and cryo-EM technology to obtain insight into the structural basis of the 25HC-dependent transmembrane interaction between human Scap and Insig-2.

\section{Results}

\section{Structure of the 25HC-bound Scap/Insig-2 complex}

The 25HC-dependent interaction between recombinant Scap and Insig-2 protein has been demonstrated using the insect cell-based pull-down assay, in which mouse Scap was used because of its higher expression level than the human ortholog (27). For the present study, the Scap and Insig variants used for binding assays were generated in the context of mouse Scap (residues 1-741) and full-length (FL) human Insig-2 (fig. S1B).

To obtain proteins for structural analysis, we tested a large number of constructs from different homologs with distinct boundaries, point mutations, fusion proteins, and affinity tags. Briefly, the combination of WD40 domain-deleted human Scap (residues 1-752 with N-terminal FLAG tag) and FL human Insig-2 (N-terminal His Ho $_{10}$ tag, Bril fusion, and three point mutations, C14S, C90S, and C215S) yielded sufficient complex for structural studies when transiently co-expressed in HEK293F cells supplemented with $25 \mathrm{HC}$ at a final concentration of $1 \mu \mathrm{g} / \mathrm{mL}$ in the medium. For cryo-EM analysis, proteins were extracted from the membrane using $1 \%(\mathrm{w} / \mathrm{v})$ lauryl maltose neopentyl glycol (LMNG) and $0.1 \mathrm{mg} / \mathrm{mL}$ $25 \mathrm{HC}$. After tandem affinity purification and size exclusion chromatography (SEC), peak fractions were pooled and further concentrated for cryo-EM sample preparation (fig. S1C). If $25 \mathrm{HC}$ was omitted during extraction, the complex fell apart.

Cryo-EM data processing of the complex was challenging because of the small size of the TM region - 14 TMs without symmetry ( 8 from Scap and 6 from Insig-2) - and the flexibility of the soluble domain. Combining Relion, CryoSparc, and the "guided multi-reference 3D classification" method developed by C. Yan (42), a 3D reconstruction was obtained at an overall resolution of $4.2 \AA$ (Fig. 1B). Refinement of a softmasked TM region improved the resolution to $3.7 \AA$, while the soluble domain was only poorly resolved, likely owing to its flexibility (Fig. 1C, figs. S2 and S3, and table S1).

The structural model was only built for the transmembrane domains based on the 3.7 A-resolution map. In total, 13 TMs were resolved. Using the structures of MvINS and NPC1SSD (PDB codes: 4XU6 and 6W5S, respectively) as references, the 6 TMs in Insig-2 and the first 6 TMs in Scap (Fig. 1D and fig. S3A) were unambiguously assigned. The one additional resolved TM is adjacent to TM1 in Scap. The resolution only supported modelling of poly-Ala, insufficient to distinguish whether this is TM7 or TM8. To facilitate structural illustration, we name the TMs in Insig-2 TM1-6, and those in Scap S1-S7.

Apart from the $13 \mathrm{TM}$ segments, an elongated density reminiscent of a sterol was resolved on the interface between SSD and Insig-2. Because $25 \mathrm{HC}$ is indispensable for complex formation, we assigned a $25 \mathrm{HC}$ molecule into this density (Fig. 1C, right). Below, we describe individual structures of Scap and Insig-2 before analysis of the molecular basis for 25HC-dependent protein interaction.

\section{A discontinuous S4 in Scap-SSD}

In total, 180 side chains were assigned to the TMD of Scap. S1 is preceded by a membrane-attaching transverse helix, named TH1 (residues 5-25), and the last resolved residues are ${ }_{447}$ MELA $_{450}$ in the "MELADL" motif, marking the cytosolic end of S6 (19) (Fig. 2A and fig. S4A).

The resolved TM fold of Scap is similar to that of NPC1 and Ptch1, with the root-mean-square deviation (RMSD) values of $2.4 \AA$ and $1.9 \AA$ over 129 and 127 aligned $\mathrm{C} \alpha$ atoms, respectively (Fig. 2, B and C). However, Scap has a distinct feature - the S4 segment is broken in the middle, resulting in two half helices, designated S4a and S4b (Fig. 1C, Fig. 2A, and fig. S3A). In both NPC1 and Ptch1, the corresponding segment is an intact $\alpha$ helix (Fig. 2, B and C). Because of this kink, S4a leans toward the interior of the SSD, creating space for ligand accommodation (Fig. 1C and Fig. 2, A and B). This binding site, constituted by the luminal halves of S4-S6, is on the opposite side to the S2-S4 site in Ptch1 and NPC1L1. In Scap, the corresponding S2-S4 site is shielded by the S7 (or S8) segment, which is missing in the other SSD-containing proteins of known structures (Fig. 2C).

Among the well-characterized loss or gain of function (LOF or GOF) Scap mutations, four loci are resolved in our 3D EM reconstruction (Fig. 2D). Asp428, whose Ala substitution (D428A) confers binding to Insig in the absence of sterol (43), and Tyr298, whose replacement by Cys (Y298C) led to loss of Insig binding $(13,44)$, are positioned in the middle of S6 and S2, respectively; their side chains pointing to the unwound region of S4 from opposite sides. The other two LOF $(\mathrm{L} 315 \mathrm{~F}$ and $\mathrm{D} 443 \mathrm{~N})$ loci $(6,13)$ are close to each other on the cytosolic termini of S3 and S6, respectively (Fig. 2D). 


\section{A hydrophobic pocket in Insig-2}

The resolved Insig- 2 contains residues 18-214, with the N-terminal Bril unresolved (Fig. 1B). TMs $1 / 2$ and TMs $5 / 6$ are related to each other by an approximately $180^{\circ}$ rotation around an axis that is perpendicular to the membrane. TM3 and TM4, the counterparts of which mediate trimerization of MvINS, form an anti-parallel hairpin on one side of TM6. There are some shifts of the TM boundaries from predicted models, and TM3 and TM4 are one or two helical turns shorter than a regular transmembrane $\alpha$ helix (Fig. 3A and fig. S4B).

The overall structure of Insig-2 can be superimposed onto MvINS with RMSD of $3.3 \AA$ over $141 \mathrm{C} \alpha$ atoms (Fig. 3B). The intervening sequences between TM1 and TM2 and between TM5 and TM6, designated L1-2 and L5-6, respectively, in Insig-2 are much longer than the MvINS counterparts, which form anti-parallel $\beta$-strands that cap the central cavity (Fig. $3 \mathrm{~B})$. We modeled the two linkers in the structure to indicate their length, but the sequences of residues 55-69 and residues 176-189 were barely visible in the EM map (fig. S3B).

In the crystal structure of MvINS, an endogenous diacylglycerol (DAG) molecule inserts one tail into the central pocket enclosed by TMs $1 / 2 / 3 / 5$ and projects the other into the membrane through the cleft between TM2 and TM5 (27). Based on the DAG-bound MvINS structure, the central pocket in a homologous model of Insig-2 was predicted to be the accommodation site for sterols (27). Consistent with this model, replacement of a pocket-facing residue Phe115 by Ala led to resistance to $25 \mathrm{HC}$-dependent inhibition of SREBP cleavage and abolished Scap binding in the presence of $25 \mathrm{HC}(27,40)$. Although the structure of Insig-2 has a similar central pocket and a lateral cleft, no density that may correspond to a sterol is observed within the pocket even when the map is pass-filtered to low resolutions (fig. S3B). Instead, $25 \mathrm{HC}$ binds at the interface between Scap and Insig-2. Phe115, which interacts with a number of hydrophobic residues in the central pocket, is positioned on the back of the $25 \mathrm{HC}$ binding site on TM3 of Insig-2 (Fig. 3C). Replacement of the bulky Phe by Ala may disrupt the local interactions and lead to dislocation of TM3, hence altering the $25 \mathrm{HC}$ binding site.

To corroborate that the central pocket of Insig is not the binding site for $25 \mathrm{HC}$, we conducted a series of structureguided mutational characterizations. Insig was reported to bind to $25 \mathrm{HC}$, but not cholesterol (40). Since the only difference between the two sterol molecules is the presence of a polar hydroxyl group at the end of the octyl tail of $25 \mathrm{HC}$, it is reasonable to expect that one or more polar groups in Insig would specifically recognize the $25-\mathrm{OH}$ group, should the central pocket be the accommodation site. However, the central pocket is highly hydrophobic, with only one polar residue, Ser81, nearby, but none inside (Fig. 3C). If Ser81 were the coordinating residue for the $25-\mathrm{OH}$ of $25 \mathrm{HC}$, then an Ala substitution of this locus might impair $25 \mathrm{HC}$ response. To test this, we introduced a single point mutation S81A. The variant behaved just like WT protein in both 25HCdependent Scap binding (Fig. 3D, lanes 1 to 4, and fig. S5A) and in the suppression of SREBP cleavage (Fig. 3E, lanes 1 to 6 , and fig. S5B), thus excluding Ser81 as the $25-\mathrm{OH}$ coordinating residue. An entirely hydrophobic pocket should not prefer $25 \mathrm{HC}$ to cholesterol.

If the pocket is not responsible for $25 \mathrm{HC}$ binding, blockage of the central pocket by bulky residues should have no effect on the 25HC-dependent complex formation. Based on the structure, two Insig-2 variants were generated, each containing a pair of point mutations that replaced small or linear side chains at similar depth within the pocket with Phe, L42F/C77F and L46F/V73F (Fig. 3C). There is no significant difference between these mutants and WT Insig-2 with regard to 25HC-dependent Scap interaction (Fig. 3D, lanes 1, 2, and 5 to 8 , and fig. S5A) or SREBP cleavage suppression (Fig. $3 \mathrm{E}$, lanes 1 to 3 and 7 to 12, and fig. S5B).

Taken together, our structural analysis and biochemical evidence suggests that the central pocket of Insig is not the accommodation site for the $25 \mathrm{HC}$ molecule that is required for the interaction between Scap and Insig.

\section{HC sandwiched by Scap-SSD and Insig-2}

The interaction between Scap and Insig-2 appears highly asymmetric with limited contacts. Viewed from the lumen, S2, S4, S6, and S5 of Scap form a concave surface to interact with TMs 3/4 of Insig-2 (Fig. 4A, left). Insig-2 mainly engages TM3 to pack against S2 and S4a of Scap. The luminal tip of Insig-TM1 also contacts Scap-S2 (Fig. 4A, middle, and fig. S6, $\mathrm{A}$ and $\mathrm{B})$. On the other side within the membrane, the interaction is mainly mediated through the bound $25 \mathrm{HC}$ molecule (Fig. 4A, right).

The resolved density allowed for discrimination of the sterol backbone, which is also featured with the two bulges corresponding to the $\mathrm{C} 18$ and $\mathrm{C} 19$ groups, from the linear tail of $25 \mathrm{HC}$, leaving the $3 \beta-\mathrm{OH}$ group on the luminal side (Fig. $1 \mathrm{C}$ and fig. S3C). The limited contacts between Scap and Insig result in a cytosol-facing cavity that is connected to the bound ligand (Fig. 4B). We placed the end of the iso-octanol tail adjacent to a cluster of polar and charged residues from TM3/4 of Insig- 2 and S2/S4/S5 of Scap, exposing $25-\mathrm{OH}$ to the aqueous cytosolic milieu. The hydrophilic environment in the mid-height of the membrane might explain the preference for a polar group to the hydrophobic tail of cholesterol (Fig. 4, B and $\mathrm{C}$ ).

$25 \mathrm{HC}$ is nearly parallel to the tilted S4a of Scap. The tetracyclic ring inserts into the hydrophobic pocket enclosed by S4a, S5, and S6 of Scap and TMs 3/4 of Insig-2 (Fig. 1C and Fig. 4, D and E). To validate the positioning of $25 \mathrm{HC}$ observed in the structure, we sought to introduce mutations to Scap 
and Insig residues that are implicated in $25 \mathrm{HC}$ binding but not involved in direct contact of the proteins. We propose these may disrupt the 25HC-dependent interaction between WT proteins, while allowing interaction between Insig-2 and the GOF variant Scap(D428A) in the absence of $25 \mathrm{HC}$ (43).

Among the surrounding residues, three bulky ones, Tyr351 on S4a and Ile414 and Phe417 on S6 in Scap, and two small ones, Gly117 and Ala121 on TM4 of Insig-2, together define the contour for accommodating the tetracyclic ring and the protruding $\mathrm{C} 18$ and $\mathrm{C} 19$ groups of $25 \mathrm{HC}$ (Fig. $4 \mathrm{E}$ ). However, substitution of these residues may also interfere with direct protein interaction. Based on our structure, His120 on the luminal end of TM3 of Insig-2 may contribute to the coordination of the $3 \beta-\mathrm{OH}$ group of $25 \mathrm{HC}$ via water-mediated hydrogen bonds (Fig. 4E, left). Leu343 on the loop between S3 and S4a and Val355 on S4a of Scap appear to solely interact with $25 \mathrm{HC}$. These three residues were selected for examination (Fig. 4E).

When treated with $0.1 \mu \mathrm{g} / \mathrm{mL} 25 \mathrm{HC}$, the WT Scap pulled down by Insig-2(H120A) was less than half than by WT Insig2. When L343A and V355A were individually introduced to Scap, the amount of retained Scap mutants was each less than $25 \%$ of the WT protein (Fig. 4F, lanes 1 to 4 , and figs. S7 and S8). Scap(D428A) maintains binding to Insig in the absence of $25 \mathrm{HC}$, although the interaction is stronger in the presence of $25 \mathrm{HC}$ (Fig. $4 \mathrm{~F}$, lanes 9 and 13, and figs. S7 and S8). When the three mutations were each combined with Scap(D428A), complex formation in the absence of $25 \mathrm{HC}$ was only slightly affected (Fig. 4F, lanes 13 to 16 , and figs. S7 and S8).

Consistently, these mutations alone also led to impaired 25HC-dependent suppression of SREBP cleavage. But when they were in combination with $\operatorname{Scap}(\mathrm{D} 428 \mathrm{~A})$, there was no detectable difference for suppression of SREBP activation in the absence of $25 \mathrm{HC}$ (fig. S9). Therefore, the pull-down and SREBP cleavage assays collectively consolidate the structureelucidated binding site for $25 \mathrm{HC}$.

\section{Discontinuity of Scap-S4 is critical to Insig binding}

Mid-point unwinding of S4, a distinct feature of Scap, may serve as a switch for Insig association. On the one hand, bending in the middle of Scap-S4 and the resulting tilt of S4a creates the cavity between Scap and Insig to accommodate $25 \mathrm{HC}$ (Fig. 2B and Fig. 4D). On the other hand, unwinding of ScapS4 provides the space for the bulky side chains of Arg110 on TM3 of Insig-2 and Tyr298 on S2 of Scap (Fig. 5A and fig. S11A).

A previous report showed that Insig-2(R110A) did not affect the inhibition of 25HC-dependent SREBP cleavage (40). In our pull down assay, this mutation slightly weakened 25HC-mediated association with Scap (Fig. 5B, lanes 1, 2, 5, and 6, and figs. S8 and S10A). Examination of the EM map suggested that Arg110, which was unambiguously resolved (Fig. 1C), may interact with Scap-Glu359 along with Lys102, a residue on the linker between TM3 and TM4 of Insig-2 (Fig. $5 \mathrm{~A})$. However, the side chains of Insig-Lys102 and ScapGlu359 were not well resolved. To verify their potential interactions, we generated and examined additional mutants. Whereas Insig-2(K102A) behaved similarly to WT for 25HCdependent Scap binding, E359A of Scap abolished 25HCmediated interaction with Insig-2 (Fig. 5B, lanes 3, 4, 15, and 16; fig. S8; and fig. S10, A and B). The complex could no longer form in the presence of $25 \mathrm{HC}$ when both Lys102 and Arg110 were substituted with Ala (Fig. 5B, lanes 7 and 8, and fig. S10A), suggesting redundant interaction of these two basic residues with Scap-Glu359.

An intact Scap-S4, if similar with that in other SSDcontaining proteins, would leave Insig-Arg110 in an unfavorable hydrophobic environment (fig. S11A), hence impeding interaction with Insig. Supporting a critical role of the S4 discontinuity in complex formation, when Gly357, the unwinding point, was mutated to the $\alpha$ helix-stabilizing residue Ala (45), the variant $\operatorname{Scap}(\mathrm{G} 357 \mathrm{~A})$ could not form a $25 \mathrm{HC}$ mediated complex with Insig-2 (Fig. 5B, lanes 13 and 14, and figs. S8 and S10B).

Unwinding of a TM helix in the middle would be unfavored in the hydrophobic lipid bilayer if not stabilized. Both $25 \mathrm{HC}$ binding and the nearby residues appear to affect the stability of the discontinued helix. While tilting of S4a is required for generating the sterol binding site, insertion of the sterol into the cavity can in turn stabilize the kink (Fig. 4D). In addition, Tyr298 on S2 stabilizes the unwound segment through H-bonding to the exposed backbone amide of Leu358 (Fig. 5A). Scap(Y298C) is a well-characterized LOF mutant $(44,46)$. To investigate the importance of its hydroxy group in stabilizing the S4 kink, we generated a mutant Scap(Y298F), which, similar to Y298C, failed to interact with Insig-2 in the presence of $25 \mathrm{HC}$ (Fig. $5 \mathrm{~B}$, lanes 9 to 12 , and figs. S8 and S10B).

On the other hand, S4 unwinding provides a potential explanation for the GOF of Scap(D428A). Asp428, with S4 unwound, is placed in an unfavorable environment constituted by hydrophobic residues (Fig. 5C). There is one nearby polar residue, Gln432, which is separated from Asp428 by one helical turn. In a 3.0- $\AA$ resolution structure of $\mathrm{NPC1}(36)$, the side chains of the corresponding residues, Asp771 and Gln775, interact (Fig. 5D). In Scap, however, the side group of Gln432, which points away from Asp428 to avoid steric clash with Val361, projects into an unfavorable hydrophobic surrounding (Fig. 5D). If S4 were an intact helix, Gly357 would face these two residues, leaving enough space to allow for an Asp428-interacting conformation of Gln432 (Fig. 5D).

Because of the undesirable local environment for Asp428 and Gln432 with a broken S4, neutralization of them by Ala 
may lower the energy barrier for S4 unwinding; whereas the penalty has to be compensated by $25 \mathrm{HC}$ binding for WT proteins. This analysis not only provides a molecular interpretation for the GOF of Scap(D428A), where the unwound conformation of $\mathrm{S} 4$ may be more favorable and compatible with Insig association even in the absence of $25 \mathrm{HC}$, but also predicts that Q432A may lead to GOF. Indeed, the variant Scap (Q432A) could form a complex with Insig in the absence of $25 \mathrm{HC}$, but to a lesser extent than Scap(D428A) (Fig. $5 \mathrm{E}$ and figs. S8 and S10C). Taken together, the published and our structure-derived LOF and GOF mutations support a function of S4 discontinuity in Insig binding.

\section{Discussion}

In this paper, we have limited our analyses to the TM regions of Scap and Insig-2 in the presence of $25 \mathrm{HC}$. Studies in the past decade have revealed the regulatory role of Loop1 and Loop7 of Scap in response to cholesterol (10) (Fig. 5F). Loop1 and Loop7 interact with each other in the absence of cholesterol (47); upon cholesterol binding to Loop 1 (41), the SSD undergoes conformational changes that are compatible with Insig binding and cause separation of Loop1 and Loop7 (48). The conformation of Scap in the presence of $25 \mathrm{HC}$ and/or cholesterol prevents the MELADL motif from being recognized by COPII vesicles for trafficking to the Golgi (49). These sophisticated molecular events cannot be recapitulated from one structure, especially when the soluble domains were barely resolved (Fig. 1B). Nevertheless, structural comparison of Scap and the other SSD-containing proteins, such as NPC1 and Ptch1, provides clues that may facilitate future investigation (fig. S11).

In addition to S4, the major conformational changes of SSD occur in S2 (fig. S11, A and B). The interaction between S2 and TM3 represents a major interface between Scap and Insig (Fig. 4A and fig. S6A). If the SSD of Scap adopts a similar conformation with that in NPC1 or Ptch1, S2 can no longer reach Insig (Fig. 2, B and C, and fig. S11, A and B). In the conformation of $\mathrm{NPC1}$ or Ptch1, the equivalent residue of Tyr298 on S2 is distant from the straight S4 to avoid steric clash, thus unable to stabilize the unwinding of S4 (fig. S11A). Importantly, $\mathrm{S} 2$ is directly connected to Loop1. It remains to be tested whether cholesterol-dependent conformational changes of Loop1 may be coupled to the movement of S2 and S4.

Likewise, ${ }_{447} \mathrm{MELA}_{450}$ at the end of S6 are close to S2 and the putative S7 (or S8), two TM segments that are respectively contiguous with Loop 1 and Loop 7 (Fig. 2A). It is yet to be characterized whether conformational changes of Loop 1 and/or Loop 7 may be transmitted to the shifts of S2 and S7/S8 to induce structural shifts of the MELADL motif for regulated COPII recognition.

Although shown here is a heterocomplex between monomeric Scap and Insig-2, Scap was reported to be a tetramer (39) and MvINS exists as a trimer (27). If S4 remains an integral helix in Scap alone, the Insig-facing surface has several conserved polar residues, the corresponding residues in $\mathrm{NPC} 1$ and Ptch1 are protected by the SSD-like domain from the C-terminal half of the protein (figs. S1A and S11C) $(30,32)$. Homo-oligomerization of Scap-SSD may be required to shield the polar surface from the lipid bilayer. Whether Scap and Insig alone and as a complex can form a higher order oligomer remains to be determined.

The structure of the TM domains of human Scap and Insig-2 in the presence of $25 \mathrm{HC}$ suggests the importance of an interface between Scap's SSD and Insig, which would explain the requirement of $25 \mathrm{HC}$ for complex formation. Several GOF or LOF mutations can be reasonably interpreted in light of this model. Despite the advances in the mechanistic understanding of sterol sensing by Scap and Insig, there are a number of outstanding questions as discussed above. Our present structure provides clues to addressing these questions, but a comprehensive understanding awaits further investigation, including structure determination of the lone Scap and Insig proteins.

\section{Materials and methods}

\section{Protein expression and purification}

The cDNAs of full-length human Insig-2 (accession: NM_001321329), wild type (WT) or the 3CS mutant (C14S, C90S, and C215S), and WD40-deleted human Scap (accession: NM_012235, residues 1-752) were subcloned separately into the pCAG vector. Scap was tagged with an N-terminal FLAG, and the $\mathrm{N}$ terminus of Insig-2 was tandemly fused with $10 \times$ His and Bril. Point mutations were introduced using a standard two-step PCR.

HEK 293F cells (Invitrogen) were cultured in SMM 293TI medium (Sino Biological Inc.) at $37^{\circ} \mathrm{C}$ under $5 \% \mathrm{CO}_{2}$ in a Multitron-Pro shaker (Infors, $130 \mathrm{rpm}$ ). When cell density reached $2.0 \times 10^{6}$ cells per milliliter, the cells were transiently co-transfected with two plasmids and polyethylenimine (PEI) (Polysciences). The two plasmids, $\sim 0.75 \mathrm{mg}$ each, were premixed with $3 \mathrm{mg}$ PEI in $50 \mathrm{~mL}$ fresh medium for $15 \mathrm{~min}$ before adding into $1 \mathrm{~L}$ cell culture for transfection. After 24 hours, 25-hydroxylcholesterol (25HC, Sigma) was added to the cell culture at a final concentration of $1 \mu \mathrm{g} / \mathrm{mL}$. The transfected cells were cultured for another 48 hours before harvesting.

For purification of the Scap and Insig-2 complex, the cells were collected and resuspended in the buffer containing 25 $\mathrm{mM}$ Tris (pH 8.0), $150 \mathrm{mM} \mathrm{NaCl}, 1 \%$ (w/v) LMNG (Sigma), 0.1 $\mathrm{mg} / \mathrm{mL} 25 \mathrm{HC}$, and a protease inhibitor cocktail containing aprotinin at $1.3 \mu \mathrm{g} / \mathrm{mL}$, pepstatin at $0.7 \mu \mathrm{g} / \mathrm{mL}$, and leupeptin at $5 \mu \mathrm{g} / \mathrm{mL}$. After incubation at $4^{\circ} \mathrm{C}$ for 2 hours, the solution was centrifuged at $18,000 \times \mathrm{g}$ at $4^{\circ} \mathrm{C}$ for $40 \mathrm{~min}$. The 
supernatant was loaded to the M2 resin, rinsed with the wash (W2) buffer containing $25 \mathrm{mM}$ Tris (pH 8.0), $150 \mathrm{mM} \mathrm{NaCl}$, $0.005 \% \mathrm{LMNG}$, and $0.5 \mu \mathrm{g} / \mathrm{mL} 25 \mathrm{HC}$, and eluted by W2 buffer supplemented with $0.2 \mathrm{mg} / \mathrm{mL}$ FLAG peptide. The eluent was then loaded to the Ni-NTA resin and washed with the wash (W3) buffer containing $25 \mathrm{mM}$ Tris (pH 8.0), $150 \mathrm{mM} \mathrm{NaCl}$, $0.005 \%$ LMNG, and $10 \mathrm{mM}$ imidazole, followed by elution with W3 buffer plus $290 \mathrm{mM}$ imidazole. The eluent was concentrated and applied to size exclusion chromatography (SEC, Superose 6 Increase 10/300 GL, GE Healthcare) in the buffer containing $25 \mathrm{mM}$ Tris ( $\mathrm{pH} \mathrm{8.0),} 150 \mathrm{mM} \mathrm{NaCl}$, and $0.005 \%$ LMNG.

\section{Cryo-EM sample preparation and data acquisition}

Aliquots $(3.5 \mu \mathrm{L})$ of the purified human Scap/Insig-2 complex, which was concentrated to approximately $10 \mathrm{mg} / \mathrm{mL}$, were placed on glow-discharged holey carbon grids (Quantifoil $\mathrm{Au}$ R1.2/1.3, 300 mesh). After blotting for $3.0 \mathrm{~s}$, the grids were plunge-frozen in liquid ethane cooled by liquid nitrogen in Vitrobot (Mark IV, Thermo Fisher Scientific). The cryogrids were transferred to a Titan Krios operating at $300 \mathrm{kV}$ equipped with Gatan K3 Summit detector and a GIF Quantum energy filter. Micrographs were recorded in the superresolution mode with a normal magnification of $81,000 \mathrm{x}$, resulting in a calibrated pixel size of $0.544 \AA$. Each stack of 32 frames was exposed for $8 \mathrm{~s}$, with an exposing time of $0.25 \mathrm{~s}$ per frame. The total dose rate was about 5.3 counts/sec/physical-pixel $\left(\sim 4.7 \mathrm{e} / \mathrm{sec} / \AA^{2}\right)$ for each stack. AutoEMation was used for the fully automated data collection (50). All 32 frames in each stack were aligned and summed using the whole-image motion correction program MotionCor2 (51) and binned to a pixel size of $1.09 \AA$. The defocus value of each image was set to 1.5-2.0 $\mu \mathrm{m}$ and determined by Gctf (52).

\section{Data processing}

Out of 8,549 micrographs, 5,648,776 particles were automatically picked by Gautomatch (www2.mrclmb.cam.ac.uk/kzhang/Gautomatch/). Two rounds of $2 \mathrm{D}$ classification were performed using RELION $3.0(53,54)$ (fig. S2, A and B), and a small subset of the selected particles was used to generate the initial model by RELION 3.0. A good reference of low resolution and five bad references were generated using conventional 3D classification to perform the nextstep "guided multi-reference classification" following a reported procedure (42). One round of guided multi-reference classification was performed, resulting in 606,502 particles (fig. S2B). After two additional rounds of multi-reference classification of the same 606,502 particles with a pixel size of $2.18 \AA, 216,819$ particles were selected, and then re-centered and re-extracted with a pixel size of $1.09 \AA$ (fig. S2B).These particles were further processed with CRYOSPARC (55). After two additional rounds of multi-reference classification,
153,168 resulting particles yielded a reconstruction at an average resolution of $4.2 \AA$ after Non-Uniform refinement. The resolution of the TM region was improved to an average of $3.7 \AA$ after focused refinement (fig. S2, B and C, and table S1). Reported resolutions were calculated on the basis of the FSC 0.143 criterion, and the FSC curves were corrected with highresolution noise substitution methods (56). Local resolution variations were estimated using CRYOSPARC $(55,57)$. The angular distributions of the particles used for the final reconstruction of the Scap/Insig complex were reasonable (fig. S2D). The resulting map displayed clear features of secondary elements and side chains for the core region of the transmembrane (TM) domains of the Scap/Insig complex (Fig. 1C and fig. S3).

\section{Model building and refinement}

The atomic coordinates of the Scap/Insig complex were generated by combining homology modelling and de novo model building. The initial structure models for the Scap-SSD domain and Insig were generated by CHAINSAW (58) using homologous structures of NPC1 (PDB code:6W5S, 28\% identity with the SSD domain of Scap) and MvINS (PDB code: 4XU6, $26 \%$ identity with human Insig-2) as template. These structures were docked into the density map and manually adjusted and re-built in COOT (59). The model for the TM region was refined against the $3.7-\AA \AA$ map using PHENIX (60) in real space with secondary structure and geometry restraints. Overfitting of the model was monitored by refining the model in one of the two independent maps from the goldstandard refinement approach and testing the refined model against the other map (61) (fig. S2E). The structure of the Scap/Insig complex was validated through examination of the Molprobity scores (62) and statistics of the Ramachandran plots (table $\mathrm{S} 1$ ).

\section{Pull-down of Scap by Insig-2}

The pull-down assay was performed following our published protocol (27). Human Insig-2, WT or indicated variants, with an N-terminal $\mathrm{His}_{10}$-tag and WD40-deleted mouse Scap (TMD, residues 1-741), WT or indicated variants, with an Nterminal FLAG tag were co-expressed using baculovirus-infected Sf-9 insect cells, which were cultured in the SF-900 II medium (Gibco) supplemented with 100 units/mL penicillin and $100 \mu \mathrm{g} / \mathrm{mL}$ streptomycin at $27^{\circ} \mathrm{C}$. Where indicated, $25 \mathrm{HC}$ was added to the culture at final concentrations of $1 \mu \mathrm{g} / \mathrm{mL}$ or $0.1 \mu \mathrm{g} / \mathrm{mL} 24$ hours after infection. Otherwise, $25 \mathrm{HC}$ was omitted throughout the experiments. Cells were collected and resuspended in the TBS buffer (25 mM Tris ( $\mathrm{pH} 8.0)$ and 150 $\mathrm{mM} \mathrm{NaCl}$ ) plus protease inhibitor cocktail, 1\% LMNG, and $0.1 \%(\mathrm{w} / \mathrm{v})$ cholesteryl hemisuccinate Tris salt (CHS) 40 hours after infection. $25 \mathrm{HC}$ was added at indicated concentrations where it was required. After incubation at $4^{\circ} \mathrm{C}$ for 1 hour, the 
mixture was applied to centrifugation at $18,000 \times \mathrm{g}$ at $4{ }^{\circ} \mathrm{C}$ for $30 \mathrm{~min}$ and the supernatant was applied to Ni-NTA. The resin was washed with $1 \mathrm{ml}$ TBS buffer plus $30 \mathrm{mM}$ imidazole, $0.01 \%$ LMNG, and $25 \mathrm{HC}$ at indicated concentrations for seven times. The proteins were eluted using wash buffer plus $300 \mathrm{mM}$ imidazole, applied to 12\% NuPAGE (Invitrogen), and analyzed by Western blot.

For Western blotting, the proteins were transferred to Immobilon-P transfer membranes (Millipore) after SDS-PAGE. The membranes were blocked by $5 \%(\mathrm{w} / \mathrm{v})$ Bovine Serum Albumin (Amresco) at room temperature for 1 hour and incubated with the primary antibodies - anti-His tag mouse monoclonal antibody (CWBIO) for Insig-2 or anti-Flag mouse monoclonal antibody (CWBIO) for Scap - at $4^{\circ} \mathrm{C}$ overnight with a 1:2000 dilution. Then the membranes were washed with the TBST that contained $25 \mathrm{mM}$ Tris (pH 8.0), $150 \mathrm{mM}$ $\mathrm{NaCl}$, and $0.05 \%(\mathrm{w} / \mathrm{v})$ Tween-20 for four times. The bound antibodies were visualized by chemiluminescence (SuperSignal West Dura Extended Duration Substrate; Thermo) using a 1:3000 dilution of goat anti-mouse IgG antibody, Horseradish Peroxidase (HRP) conjugates, as the secondary antibody (CWBIO). Membranes were exposed by ChemiDOC XRS+ System (Bio-Rad). For semiquantitative analysis shown in Fig. $4 \mathrm{~F}$, band intensities were measured by densitometry using the software ImageJ.

\section{SREBP-2 cleavage assay}

On day 0, SRD-13A cells, a Scap-deficient cell line derived from CHO-7 cells (63), were distributed in 6-well plates at a density of $2.5 \times 10^{5}$ cells per well in medium A that contained DMEM/F12 (1:1, Gibco) supplemented with 5\% newborn calf serum (Gibco), $5 \mu \mathrm{g} / \mathrm{ml}$ cholesterol (Sigma), $1 \mathrm{mM}$ sodium mevalonolactone (Sigma), and $20 \mu \mathrm{M}$ sodium oleate (Sigma). After incubation at $37^{\circ} \mathrm{C}$ for 1-2 hours, cells in each well were transfected with plasmids as following: $0.8 \mu \mathrm{g}$ pCMV $-3 \times \mathrm{Myc}-$ mScap (mouse) variants, $0.9 \mu \mathrm{g}$ pCMV-His-hInsig-2 (human) variants, and 0.09 ug pCMV-3×Flag-hSREBP2 (human) by ViaFect ${ }^{\mathrm{TM}}$ transfection reagent (Promega). The medium was removed 16-20 hours later, and cells were washed twice with PBS that contained $135 \mathrm{mM} \mathrm{NaCl}, 4.7 \mathrm{mM} \mathrm{KCl}, 10 \mathrm{mM}$ $\mathrm{Na}_{2} \mathrm{HPO}_{4}$, and $2 \mathrm{mM} \mathrm{NaH}_{2} \mathrm{PO}_{4}(\mathrm{pH} 7.3 \pm 0.1)$ before changing for medium $\mathrm{B}$ (medium A supplemented with 5\% lipoproteindeficient serum, $50 \mu \mathrm{M}$ mevastatin, and $50 \mu \mathrm{M}$ sodium mevalonolactone) plus $1 \%$ hydroxypropyl- $\beta$-cyclodextrin (Sigma). After incubation at $37^{\circ} \mathrm{C}$ for 1 hour, cells were washed twice with PBS and switched to medium B in the absence or presence of 25-HC. Protease inhibitors, $1.5 \mu \mathrm{M}$ FMKZ-VAD (MedChemExpress) and $20 \mu \mathrm{g} / \mathrm{mL} \mathrm{N}$-acetyl-leucinalleucinal-norleucinal (ALLN) (MedChemExpress), were added to prevent degradation of the $\mathrm{N}$-terminal fragment of SREBP2. After incubation for another 4-5 hours at $37^{\circ} \mathrm{C}$, cells were washed twice with cold PBS and subsequently suspended in
$100 \mu \mathrm{L}$ lysis buffer (PBS supplemented with 0.5\% SDS, protease inhibitors, and benzonase). The cell lysates were left on a shaker at $4^{\circ} \mathrm{C}$ for $15 \mathrm{~min}$. The total protein concentration of each cell lysate was determined by UV280 using Nanodrop. The cell lysates were mixed with $5 \times$ sample buffer $(250 \mathrm{mM}$ Tris- $\mathrm{HCl}$ (pH 6.8), 10\% SDS, 50\% glycerol, $0.2 \%$ bromphenol blue, and $500 \mathrm{mM}$ dithiothreitol). Then an aliquot (10 20 $\mu \mathrm{g}$ total protein) of each sample was loaded to NuPAGE ${ }^{\mathrm{TM}} 4-12 \%$ Bis-Tris gradient Gel (Invitrogen). After electrophoresis, the proteins were transferred to Hybond-C nitrocellulose membranes (Bio-Rad). Strips were blocked with $4 \%$ (w/v) milk in TBST, and incubated with primary antibodies for 1 hour at room temperature. The following antibodies were used: anti His-tag mouse monoclonal antibody (CWBIO), anti-Flag-tag mouse monoclonal antibody (MBL International), and anti cMyc tag mouse monoclonal antibody (9E10, Invitrogen). After incubation with the primary antibodies, the washed membranes were incubated with HRP conjugated goat anti-mouse secondary antibodies (CWBIO) at room temperature for $1 \mathrm{~h}$. Membranes were exposed by ChemiDOC XRS+ System (BioRad). The band intensities of precursor (P) and nuclear (N) form of SREBP2 were measured by densitometry using the software ImageJ. Resulting data were analyzed by GraphPad Prism 7.00. The mean ratio of the cleaved form to total SREBP2 $(\mathrm{N} /(\mathrm{N}+\mathrm{P}))$ for WT proteins in the absence of $25 \mathrm{HC}$ in three experiments was set as 1 , against which the ratio of other groups was normalized.

\section{Circular dichroism (CD) characterization of purified protein}

The recombinant $\mathrm{N}$-terminal $\mathrm{His}_{10}$-tagged human Insig-2 variants and the transmembrane domain of N-terminal FLAGtagged mouse Scap variants were individually expressed in Sf-9 insect cells in the SF-900 II medium (Gibco) supplemented with 100 units/mL penicillin and $100 \mu \mathrm{g} / \mathrm{mL}$ streptomycin at $27^{\circ} \mathrm{C}$. 48 hours after infection, cells were collected and resuspended in the TBS buffer containing $25 \mathrm{mM}$ Tris (pH 8.0) and $150 \mathrm{mM} \mathrm{NaCl}$, the protease inhibitor cocktail, $1 \% \mathrm{LMNG}$, and $0.1 \% \mathrm{CHS}$. After incubation at $4^{\circ} \mathrm{C}$ for 2 hours, the mixture was centrifuged at $18,000 \times \mathrm{g}$ at $4^{\circ} \mathrm{C}$ for $30 \mathrm{~min}$. For Insig-2 variants, the supernatant was applied to Ni-NTA, which was then washed with the TBS buffer plus $30 \mathrm{mM}$ imidazole, $0.01 \%$ LMNG, and $0.001 \%$ CHS. The proteins were eluted using wash buffer plus $300 \mathrm{mM}$ imidazole. For Scap variants, the supernatant was applied to anti-FLAG M2 affinity resin, which was washed with the TBS buffer plus $0.01 \%$ LMNG and $0.001 \%$ CHS. The proteins were eluted using wash buffer plus $0.2 \mathrm{mg} / \mathrm{mL}$ FLAG peptide. The eluent was then concentrated and applied to SEC (Superose 6 Increase 10/300 GL, GE Healthcare) in the buffer containing $25 \mathrm{mM}$ Tris (pH 8.0), $150 \mathrm{mM} \mathrm{NaCl}, 0.01 \%$ LMNG, and $0.001 \%$ CHS. The peak fractions were pooled and reinjected to SEC. The peak 
fractions from the second-round SEC were concentrated and diluted for final buffer condition as $25 \mathrm{mM}$ Tris ( $\mathrm{pH} 8.0$ ), 10 $\mathrm{mM} \mathrm{NaCl}, 0.01 \%$ LMNG, and 0.001\% CHS. The circular dichroism spectra of proteins at $\sim 0.1 \mathrm{mg} / \mathrm{mL}$ were carried out using Chirascan plus (Applied Photophysics) at room temperature.

\section{REFERENCES AND NOTES}

1. M. S. Brown, J. L. Goldstein, The SREBP pathway: Regulation of cholesterol metabolism by proteolysis of a membrane-bound transcription factor. Cell 89 , 331-340 (1997). doi:10.1016/S0092-8674(00)80213-5 Medline

2. J. L. Goldstein, R. A. DeBose-Boyd, M. S. Brown, Protein sensors for membrane sterols. Cell 124, 35-46 (2006). doi:10.1016/j.cell.2005.12.022 Medline

3. C. Yokoyama, X. Wang, M. R. Briggs, A. Admon, J. Wu, X. Hua, J. L. Goldstein, M. S. Brown, SREBP-1, a basic-helix-loop-helix-leucine zipper protein that controls transcription of the low density lipoprotein receptor gene. Cell 75, 187-197 (1993). doi:10.1016/S0092-8674(05)80095-9 Medline

4. X. Hua, C. Yokoyama, J. Wu, M. R. Briggs, M. S. Brown, J. L. Goldstein, X. Wang, SREBP-2, a second basic-helix-loop-helix-leucine zipper protein that stimulates transcription by binding to a sterol regulatory element. Proc. Natl. Acad. Sci. U.S.A. 90, 11603-11607 (1993). doi:10.1073/pnas.90.24.11603 Medline

5. X. Wang, R. Sato, M. S. Brown, X. Hua, J. L. Goldstein, SREBP-1, a membrane-bound transcription factor released by sterol-regulated proteolysis. Cell 77, 53-62 (1994). doi:10.1016/0092-8674(94)90234-8 Medline

6. X. Hua, A. Nohturfft, J. L. Goldstein, M. S. Brown, Sterol resistance in $\mathrm{CHO}$ cells traced to point mutation in SREBP cleavage-activating protein. Cell 87, 415-426 (1996). doi:10.1016/S0092-8674(00)81362-8 Medline

7. J. Sakai, A. Nohturfft, D. Cheng, Y. K. Ho, M. S. Brown, J. L. Goldstein, Identification of complexes between the $\mathrm{COOH}$-terminal domains of sterol regulatory elementbinding proteins (SREBPs) and SREBP cleavage-activating protein. J. Biol. Chem. 272, 20213-20221 (1997). doj:10.1074/ibc.272.32.20213 Medline

8. A. Nohturfft, M. S. Brown, J. L. Goldstein, Topology of SREBP cleavage-activating protein, a polytopic membrane protein with a sterol-sensing domain. J. Biol. Chem. 273, 17243-17250 (1998). doi:10.1074/jbc.273.27.17243 Medline

9. T. Yang, J. L. Goldstein, M. S. Brown, Overexpression of membrane domain of SCAP prevents sterols from inhibiting SCAP.SREBP exit from endoplasmic reticulum. J. Biol. Chem. 275, 29881-29886 (2000). doi:10.1074/ibc.M005439200 Medline

10. M. S. Brown, A. Radhakrishnan, J. L. Goldstein, Retrospective on Cholesterol Homeostasis: The Central Role of Scap. Annu. Rev. Biochem. 87, 783-807 (2018). doi:10.1146/annurev-biochem-062917-011852 Medline

11. T. Yang, P. J. Espenshade, M. E. Wright, D. Yabe, Y. Gong, R. Aebersold, J. L. Goldstein, M. S. Brown, Crucial step in cholesterol homeostasis: Sterols promote binding of SCAP to INSIG-1, a membrane protein that facilitates retention of SREBPs in ER. Cell 110, 489-500 (2002). doi:10.1016/S0092-8674(02)00872-3 Medline

12. D. Yabe, M. S. Brown, J. L. Goldstein, Insig-2, a second endoplasmic reticulum protein that binds SCAP and blocks export of sterol regulatory element-binding proteins. Proc. Natl. Acad. Sci. U.S.A. 99, $12753-12758$ (2002). doi:10.1073/pnas.162488899 Medline

13. D. Yabe, Z. P. Xia, C. M. Adams, R. B. Rawson, Three mutations in sterol-sensing domain of SCAP block interaction with insig and render SREBP cleavage insensitive to sterols. Proc. Natl. Acad. Sci. U.S.A. 99, 16672-16677 (2002). doi:10.1073/pnas.262669399 Medline

14. C. M. Adams, J. Reitz, J. K. De Brabander, J. D. Feramisco, L. Li, M. S. Brown, J. L. Goldstein, Cholesterol and 25-hydroxycholesterol inhibit activation of SREBPs by different mechanisms, both involving SCAP and Insigs. J. Biol. Chem. 279, 5277252780 (2004). doi:10.1074/jbc.M410302200 Medline

15. J. Sakai, E. A. Duncan, R. B. Rawson, X. Hua, M. S. Brown, J. L. Goldstein, Sterolregulated release of SREBP-2 from cell membranes requires two sequential cleavages, one within a transmembrane segment. Cell 85, 1037-1046 (1996). doi:10.1016/S0092-8674(00)81304-5 Medline

16. R. B. Rawson, N. G. Zelenski, D. Nijhawan, J. Ye, J. Sakai, M. T. Hasan, T. Y. Chang, M. S. Brown, J. L. Goldstein, Complementation cloning of S2P, a gene encoding a putative metalloprotease required for intramembrane cleavage of SREBPs. Mol. Cell 1, 47-57 (1997). doi:10.1016/S1097-2765(00)80006-4 Medline

17. J. Sakai, R. B. Rawson, P. J. Espenshade, D. Cheng, A. C. Seegmiller, J. L. Goldstein, M. S. Brown, Molecular identification of the sterol-regulated luminal protease that cleaves SREBPs and controls lipid composition of animal cells. Mol. Cell 2, 505514 (1998). doj:10.1016/S1097-2765(00)80150-1 Medline

18. P. J. Espenshade, W. P. Li, D. Yabe, Sterols block binding of COPIl proteins to SCAP, thereby controlling SCAP sorting in ER. Proc. Natl. Acad. Sci. U.S.A. 99, 1169411699 (2002). doi:10.1073/pnas.182412799 Medline

19. L. P. Sun, L. Li, J. L. Goldstein, M. S. Brown, Insig required for sterol-mediated inhibition of Scap/SREBP binding to COPII proteins in vitro. J. Biol. Chem. 280, 26483-26490 (2005). doi:10.1074/jbc.M504041200 Medline

20. J. R. Smith, T. F. Osborne, J. L. Goldstein, M. S. Brown, Identification of nucleotides responsible for enhancer activity of sterol regulatory element in low density lipoprotein receptor gene. J. Biol. Chem. 265, 2306-2310 (1990). doi:10.1016/S0021-9258(19)39976-4 Medline

21. J. R. Smith, T. F. Osborne, M. S. Brown, J. L. Goldstein, G. Gil, Multiple sterol regulatory elements in promoter for hamster 3-hydroxy-3-methylglutarylcoenzyme A synthase. J. Biol. Chem. 263, 18480-18487 (1988). Medline

22. M. S. Brown, J. L. Goldstein, A proteolytic pathway that controls the cholesterol content of membranes, cells, and blood. Proc. Natl. Acad. Sci. U.S.A. 96, 1104111048 (1999). doi:10.1073/pnas.96.20.11041 Medline

23. N. Sever, B.-L. Song, D. Yabe, J. L. Goldstein, M. S. Brown, R. A. DeBose-Boyd, Insig-dependent ubiquitination and degradation of mammalian 3-hydroxy-3methylglutaryl-CoA reductase stimulated by sterols and geranylgeraniol. J. Biol. Chem. 278, 52479-52490 (2003). doi:10.1074/jbc.M310053200 Medline

24. B. L. Song, N. Sever, R. A. DeBose-Boyd, Gp78, a membrane-anchored ubiquitin ligase, associates with Insig-1 and couples sterol-regulated ubiquitination to degradation of HMG CoA reductase. Mol. Cell 19, 829-840 (2005). doi:10.1016/i.molcel.2005.08.009 Medline

25. Y. Jo, R. A. Debose-Boyd, Control of cholesterol synthesis through regulated ERassociated degradation of HMG CoA reductase. Crit. Rev. Biochem. Mol. Biol. 45, 185-198 (2010). doi:10.3109/10409238.2010.485605 Medline

26. L. Feng, H. Yan, Z. Wu, N. Yan, Z. Wang, P. D. Jeffrey, Y. Shi, Structure of a site-2 protease family intramembrane metalloprotease. Science 318, 1608-1612 (2007). doi:10.1126/science.1150755 Medline

27. R. Ren, X. Zhou, Y. He, M. Ke, J. Wu, X. Liu, C. Yan, Y. Wu, X. Gong, X. Lei, S. F. Yan, A. Radhakrishnan, N. Yan, Crystal structure of a mycobacterial Insig homolog provides insight into how these sensors monitor sterol levels. Science 349, 187191 (2015). doi:10.1126/science.aab1091 Medline

28. X. Gong, J. Li, W. Shao, J. Wu, H. Qian, R. Ren, P. Espenshade, N. Yan, Structure of the WD40 domain of SCAP from fission yeast reveals the molecular basis for SREBP recognition. Cell Res. 25, 401-411 (2015). doi:10.1038/cr.2015.32 Medline

29. X. Gong, H. Qian, W. Shao, J. Li, J. Wu, J.-J. Liu, W. Li, H.-W. Wang, P. Espenshade, N. Yan, Complex structure of the fission yeast SREBP-SCAP binding domains reveals an oligomeric organization. Cell Res. 26, 1197-1211 (2016). doj:10.1038/cr.2016.123 Medline

30. X. Gong, H. Qian, X. Zhou, J. Wu, T. Wan, P. Cao, W. Huang, X. Zhao, X. Wang, P. Wang, Y. Shi, G. F. Gao, Q. Zhou, N. Yan, Structural Insights into the Niemann-Pick C1 (NPC1)-Mediated Cholesterol Transfer and Ebola Infection. Cell 165, 14671478 (2016). doi:10.1016/i.cell.2016.05.022 Medline

31. X. Li, J. Wang, E. Coutavas, H. Shi, Q. Hao, G. Blobel, Structure of human NiemannPick C1 protein. Proc. Natl. Acad. Sci. U.S.A. 113, $8212-8217$ (2016). doi:10.1073/pnas.1607795113 Medline

32. X. Gong, H. Qian, P. Cao, X. Zhao, Q. Zhou, J. Lei, N. Yan, Structural basis for the recognition of Sonic Hedgehog by human Patched1. Science 361, eaas8935 (2018). doi:10.1126/science.aas8935 Medline

33. X. Qi, P. Schmiege, E. Coutavas, J. Wang, X. Li, Structures of human Patched and its complex with native palmitoylated sonic hedgehog. Nature 560, 128-132 (2018). doi:10.1038/s41586-018-0308-7 Medline

34. Y. Zhang, D. P. Bulkley, Y. Xin, K. J. Roberts, D. E. Asarnow, A. Sharma, B. R. Myers, W. Cho, Y. Cheng, P. A. Beachy, Structural Basis for Cholesterol Transport-like Activity of the Hedgehog Receptor Patched. Cell 175, 1352-1364.e14 (2018). doi:10.1016/i.cell.2018.10.026 Medline

35. M. B. L. Winkler, R. T. Kidmose, M. Szomek, K. Thaysen, S. Rawson, S. P. Muench, 
D. Wüstner, B. P. Pedersen, Structural Insight into Eukaryotic Sterol Transport through Niemann-Pick Type C Proteins. Cell 179, 485-497.e18 (2019). doi:10.1016/i.cell.2019.08.038 Medline

36. H. Qian, X. Wu, X. Du, X. Yao, X. Zhao, J. Lee, H. Yang, N. Yan, Structural Basis of Low-pH-Dependent Lysosomal Cholesterol Egress by NPC1 and NPC2. Cell 182, 98-111.e18 (2020). doi:10.1016/i.cell.2020.05.020

37. F. Cannac, C. Qi, J. Falschlunger, G. Hausmann, K. Basler, V. M. Korkhov, Cryo-EM structure of the Hedgehog release protein Dispatched. Sci. Adv. 6, eaay7928 (2020). doi:10.1126/sciadv.aay7928 Medline

38. C.-S. Huang, X. Yu, P. Fordstrom, K. Choi, B. C. Chung, S.-H. Roh, W. Chiu, M. Zhou, X. Min, Z. Wang, Cryo-EM structures of NPC1L1 reveal mechanisms of cholesterol transport and ezetimibe inhibition. Sci. Adv. 6, eabb1989 (2020). doi:10.1126/sciadv.abb1989 Medline

39. A. Radhakrishnan, L.-P. Sun, H. J. Kwon, M. S. Brown, J. L. Goldstein, Direct binding of cholesterol to the purified membrane region of SCAP: Mechanism for a sterolsensing domain. Mol. Cell 15, 259-268 (2004). doi:10.1016/i.molcel.2004.06.019 Medline

40. A. Radhakrishnan, Y. Ikeda, H. J. Kwon, M. S. Brown, J. L. Goldstein, Sterolregulated transport of SREBPs from endoplasmic reticulum to Golgi: Oxysterols block transport by binding to Insig. Proc. Natl. Acad. Sci. U.S.A. 104, 6511-6518 (2007). doi:10.1073/pnas.0700899104 Medline

41. M. Motamed, Y. Zhang, M. L. Wang, J. Seemann, H. J. Kwon, J. L. Goldstein, M. S. Brown, Identification of luminal Loop 1 of Scap protein as the sterol sensor that maintains cholesterol homeostasis. J. Biol. Chem. 286, 18002-18012 (2011). doi:10.1074/jbc.M111.238311 Medline

42. X. Zhang, C. Yan, J. Hang, L. I. Finci, J. Lei, Y. Shi, An Atomic Structure of the Human Spliceosome. Cell 169, 918-929.e14 (2017). doi:10.1016/i.cell.2017.04.033 Medline

43. J. D. Feramisco, A. Radhakrishnan, Y. Ikeda, J. Reitz, M. S. Brown, J. L. Goldstein, Intramembrane aspartic acid in SCAP protein governs cholesterol-induced conformational change. Proc. Natl. Acad. Sci. U.S.A. 102, 3242-3247 (2005). doi:10.1073/pnas.0500206102 Medline

44. A. Nohturfft, M. S. Brown, J. L. Goldstein, Sterols regulate processing of carbohydrate chains of wild-type SREBP cleavage-activating protein (SCAP), but not sterol-resistant mutants Y298C or D443N. Proc. Natl. Acad. Sci. U.S.A. 95, 12848-12853 (1998). doi:10.1073/pnas.95.22.12848 Medline

45. E. J. Spek, C. A. Olson, Z. Shi, N. R. Kallenbach, Alanine Is an Intrinsic $\alpha$-Helix Stabilizing Amino Acid. J. Am. Chem. Soc. 121, 5571-5572 (1999). doi:10.1021/ja990056x

46. A. Roth, R. Looser, M. Kaufmann, S. M. Blättler, F. Rencurel, W. Huang, D. D. Moore, U. A. Meyer, Regulatory cross-talk between drug metabolism and lipid homeostasis: Constitutive androstane receptor and pregnane $\mathrm{X}$ receptor increase Insig-1 expression. Mol. Pharmacol. 73, 1282-1289 (2008). doi:10.1124/mol.107.041012 Medline

47. Y. Zhang, K. M. Lee, L. N. Kinch, L. Clark, N. V. Grishin, D. M. Rosenbaum, M. S. Brown, J. L. Goldstein, A. Radhakrishnan, Direct Demonstration That Loopl of Scap Binds to Loop7: A Crucial Event in Cholesterol Homeostasis. J. Biol. Chem. 291, 12888-12896 (2016). doi:10.1074/ibc.M116.729798 Medline

48. Y. Gao, Y. Zhou, J. L. Goldstein, M. S. Brown, A. Radhakrishnan, Cholesterolinduced conformational changes in the sterol-sensing domain of the Scap protein suggest feedback mechanism to control cholesterol synthesis. J. Biol. Chem. 292, 8729-8737 (2017). doi:10.1074/jbc.M117.783894 Medline

49. Y. Zhang, M. Motamed, J. Seemann, M. S. Brown, J. L. Goldstein, Point mutation in luminal loop 7 of Scap protein blocks interaction with loop 1 and abolishes movement to Golgi. J. Biol. Chem. 288, 14059-14067 (2013). doi:10.1074/jbc.M113.469528 Medline

50. J. Lei, J. Frank, Automated acquisition of cryo-electron micrographs for single particle reconstruction on an FEI Tecnai electron microscope. J. Struct. Biol. 150, 69-80 (2005). doi:10.1016/i.jsb.2005.01.002 Medline

51. S. Q. Zheng, E. Palovcak, J.-P. Armache, K. A. Verba, Y. Cheng, D. A. Agard, MotionCor2: Anisotropic correction of beam-induced motion for improved cryoelectron microscopy. Nat. Methods 14, 331-332 (2017). doi:10.1038/nmeth.4193 Medline

52. K. Zhang, Gctf: Real-time CTF determination and correction. J. Struct. Biol. 193, 112 (2016). doi:10.1016/i.jsb.2015.11.003 Medline
53. D. Kimanius, B. O. Forsberg, S. H. Scheres, E. Lindahl, Accelerated cryo-EM structure determination with parallelisation using GPUs in RELION-2. eLife 5, e18722 (2016). doi:10.7554/eLife.18722 Medline

54. S. H. Scheres, RELION: Implementation of a Bayesian approach to cryo-EM structure determination. J. Struct. Biol. 180, 519-530 (2012). doi:10.1016/i.jsb.2012.09.006 Medline

55. A. Punjani, J. L. Rubinstein, D. J. Fleet, M. A. Brubaker, cryoSPARC: Algorithms for rapid unsupervised cryo-EM structure determination. Nat. Methods 14, 290-296 (2017). doi:10.1038/nmeth.4169

56. S. Chen, G. McMullan, A. R. Faruqi, G. N. Murshudov, J. M. Short, S. H. W. Scheres, R. Henderson, High-resolution noise substitution to measure overfitting and validate resolution in $3 \mathrm{D}$ structure determination by single particle electron cryomicroscopy. Ultramicroscopy 135, 24-35 (2013). doi:10.1016/i.ultramic.2013.06.004 Medline

57. S. M. Stagg, A. J. Noble, M. Spilman, M. S. Chapman, ResLog plots as an empirical metric of the quality of cryo-EM reconstructions. J. Struct. Biol. 185, 418-426 (2014). doi:10.1016/i.jsb.2013.12.010 Medline

58. N. Stein, CHAINSAW: A program for mutating pdb files used as templates in molecular replacement. J. Appl. Cryst. 41, 641-643 (2008). doi:10.1107/S0021889808006985

59. P. Emsley, K. Cowtan, Coot: Model-building tools for molecular graphics. Acta Cryst. D60, 2126-2132 (2004). doi:10.1107/S0907444904019158 Medline

60. P. V. Afonine, B. K. Poon, R. J. Read, O. V. Sobolev, T. C. Terwilliger, A. Urzhumtsev, P. D. Adams, Real-space refinement in PHENIX for cryo-EM and crystallography. Acta Cryst. D74, 531-544 (2018). doi:10.1107/S2059798318006551 Medline

61. A. Amunts, A. Brown, X. C. Bai, J. L. Llácer, T. Hussain, P. Emsley, F. Long, G. Murshudov, S. H. W. Scheres, V. Ramakrishnan, Structure of the yeast mitochondrial large ribosomal subunit. Science 343, 1485-1489 (2014). doi:10.1126/science. 1249410 Medline

62. I. W. Davis, A. Leaver-Fay, V. B. Chen, J. N. Block, G. J. Kapral, X. Wang, L. W. Murray, W. B. Arendall 3rd, J. Snoeyink, J. S. Richardson, D. C. Richardson, MolProbity: All-atom contacts and structure validation for proteins and nucleic acids. Nucleic Acids Res. 35, W375-W383 (2007). doi:10.1093/nar/gkm216 Medline

63. R. B. Rawson, R. DeBose-Boyd, J. L. Goldstein, M. S. Brown, Failure to cleave sterol regulatory element-binding proteins (SREBPs) causes cholesterol auxotrophy in Chinese hamster ovary cells with genetic absence of SREBP cleavage-activating protein. J. Biol. Chem. 274, 28549-28556 (1999). doi:10.1074/jbc.274.40.28549 Medline

64. E. F. Pettersen, T. D. Goddard, C. C. Huang, G. S. Couch, D. M. Greenblatt, E. C. Meng, T. E. Ferrin, UCSF Chimera-A visualization system for exploratory research and analysis. J. Comput. Chem. 25, 1605-1612 (2004) doi:10.1002/jcc.20084 Medline

65. Schrodinger, LLC. (2015).

66. F. Corpet, Multiple sequence alignment with hierarchical clustering. Nucleic Acids Res. 16, 10881-10890 (1988). doi:10.1093/nar/16.22.10881 Medline

67. Y. Z. Tan, P. R. Baldwin, J. H. Davis, J. R. Williamson, C. S. Potter, B. Carragher, D. Lyumkis, Addressing preferred specimen orientation in single-particle cryo-EM through tilting. Nat. Methods 14, 793-796 (2017). doi:10.1038/nmeth.4347 Medline

\section{ACKNOWLEDGMENTS}

We thank Prof. Baoliang Song for sharing the SRD-13A cells and the plasmid pCMV$3 \times$ Flag-SREBP2. We thank the cryo-EM Facility of Westlake University and the Tsinghua University Branch of China National Center for Protein Sciences (Beijing) for providing cryo-EM and computation support. We thank Li Huang (Westlake University) and Xiaomin Li (Tsinghua University) for technical support during EM image acquisition. Funding: This work was funded by the National Key R\&D Program of China (2020YFA0509301 to C.Y.) from Ministry of Science and Technology of China, Beijing Nova Program (Z201100006820039 to C.Y.), the China Postdoctoral Science Foundation (2020M681937 to R.Y), the National Postdoctoral Program for Innovative Talents of China (BX20200304 to R.Y), and the National Natural Science Foundation of China (project 31971123 to Q.Z.). N.Y. acknowledges the Ministry of Science and Technology of China and the National 
Natural Science Foundation of China for multiple funds that have supported this research in 2008-2017. N.Y. has been supported by the Shirley M. Tilghman endowed professorship from Princeton University since 2017. Author

contributions: N.Y. conceived and supervised the project. N.Y., R.Y. P.C., W.S., H.Q., and C.Y. designed the experiments. R.Y., H.Q., X.Z., Y.L., S.G., P.C., X.G., performed molecular cloning, protein purification, and cryo-EM data acquisition. P.C., W.S., and R.Y. performed all functional assays. X.D., H.C., A.J.B. and H.Y. contributed to the design and data analysis of the SREBP cleavage assay. X.L. and J.S. generated antibodies. J.L. and Q.Z. contributed to data acquisition and processing. C.Y. performed data processing and structural building and refinement. All authors contributed to data analysis. N.Y. wrote the manuscript. Competing interests: The authors declare no competing interests. Data and materials availability: The atomic coordinates and EM map have been deposited in the Protein Data Bank (www.rcsb.org) and EMDB

(www.ebi.ac.uk/pdbe/emdb/) with the accession codes 6M49 and EMD-30074, respectively. Materials are available from the corresponding authors upon request.

\section{SUPPLEMENTARY MATERIALS}

science.sciencemag.org/cgi/content/full/science.abb2224/DC1

Figs. S1 to S11

Table S1

References $(66,67)$

MDAR Reproducibility Checklist

8 February 2020; resubmitted 31 July 2020

Accepted 6 January 2021

Published online 14 January 2021

10.1126/science.abb2224 
A
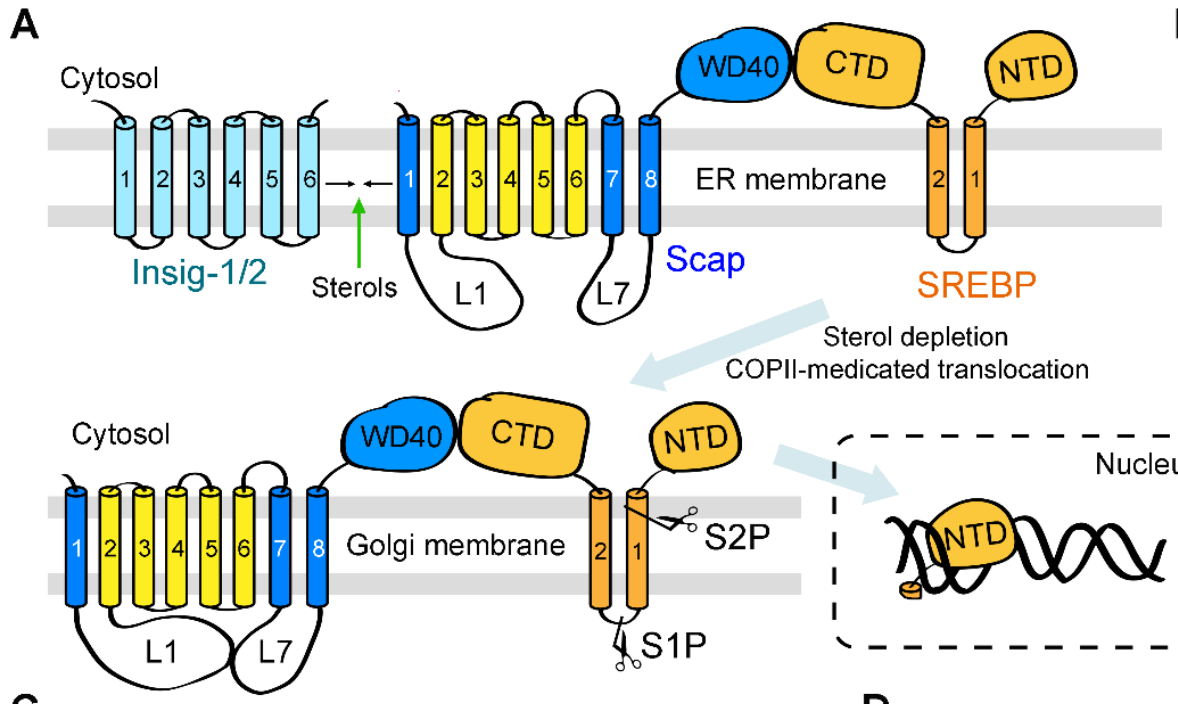

B

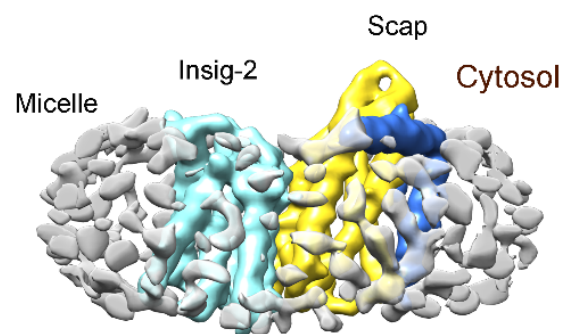

Lumen
C

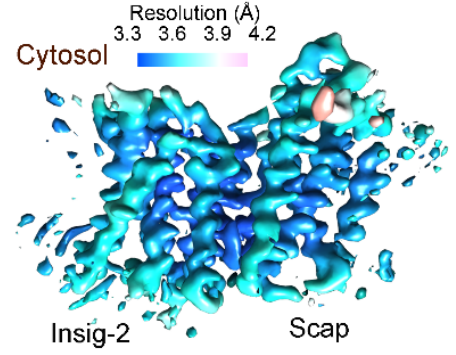

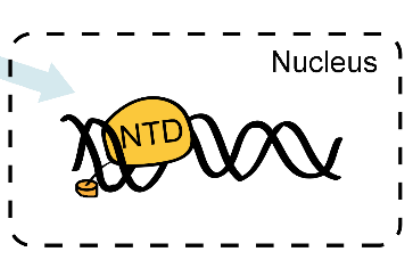

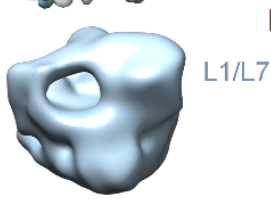

D Insig-2 Scap

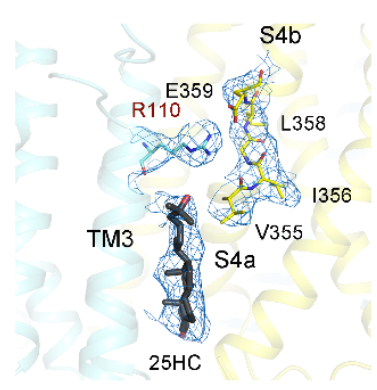

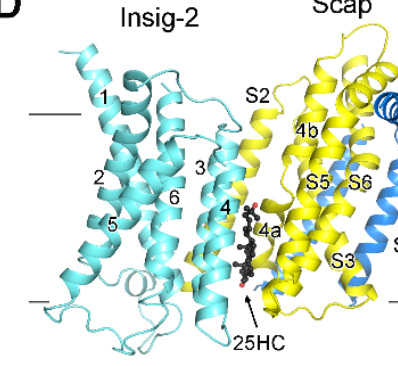

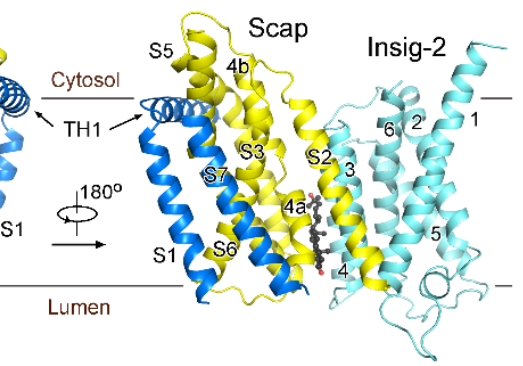

Fig. 1. Structure of the human Scap and Insig-2 complex in the presence of 25HC. (A) A simplified cartoon that illustrates several key players in the SREBP pathway. $25 \mathrm{HC}$ promotes the interaction between Scap and Insig-1/2, whereas sterol depletion leads to their dissociation and the COPII vesicle-mediated transport of the Scap and SREBP complex from the ER membrane to the Golgi. L1/L7: the luminal loops L1 and L7. NTD: the amino (N) terminal domain; CTD: the carboxy terminal domain. (B) The transmembrane (TM) region is better resolved than the soluble domains in the 3D EM reconstruction. The densities corresponding to Insig-2, the sterol sensing domain (SSD), other transmembrane (TM) segments, and the luminal domain of Scap are low pass filtered to $8 \AA$ and colored cyan, yellow, blue, and light cyan, respectively. The same color scheme is applied to all figures. The LMNG micelle is shown as gray, semi-transparent surface to indicate the orientation of the TM domain. In all figures, the cytosolic side is placed on top in the side views. Because of the low resolution of the luminal domains, we focus on the TM region for analysis in this study. The EM map was generated in Chimera (64). (C) The interface between Scap and Insig-2 and the sandwiched 25HC are clearly resolved. Left: The local densities were calculated using Relion 3.0 and presented in Chimera. Right: The densities for the ligand and surrounding key residues, shown as blue mesh, are contoured at $5 \sigma$. Note that S4 in Scap is broken in the middle, and the two half segments are designated S4a and S4b. (D) Overall structure of the TM domain of the complex. The TM segments in Insig-2 are denoted by numbers, while those in Scap are labeled S1-S7. All structure figures were prepared in PyMol (65). 

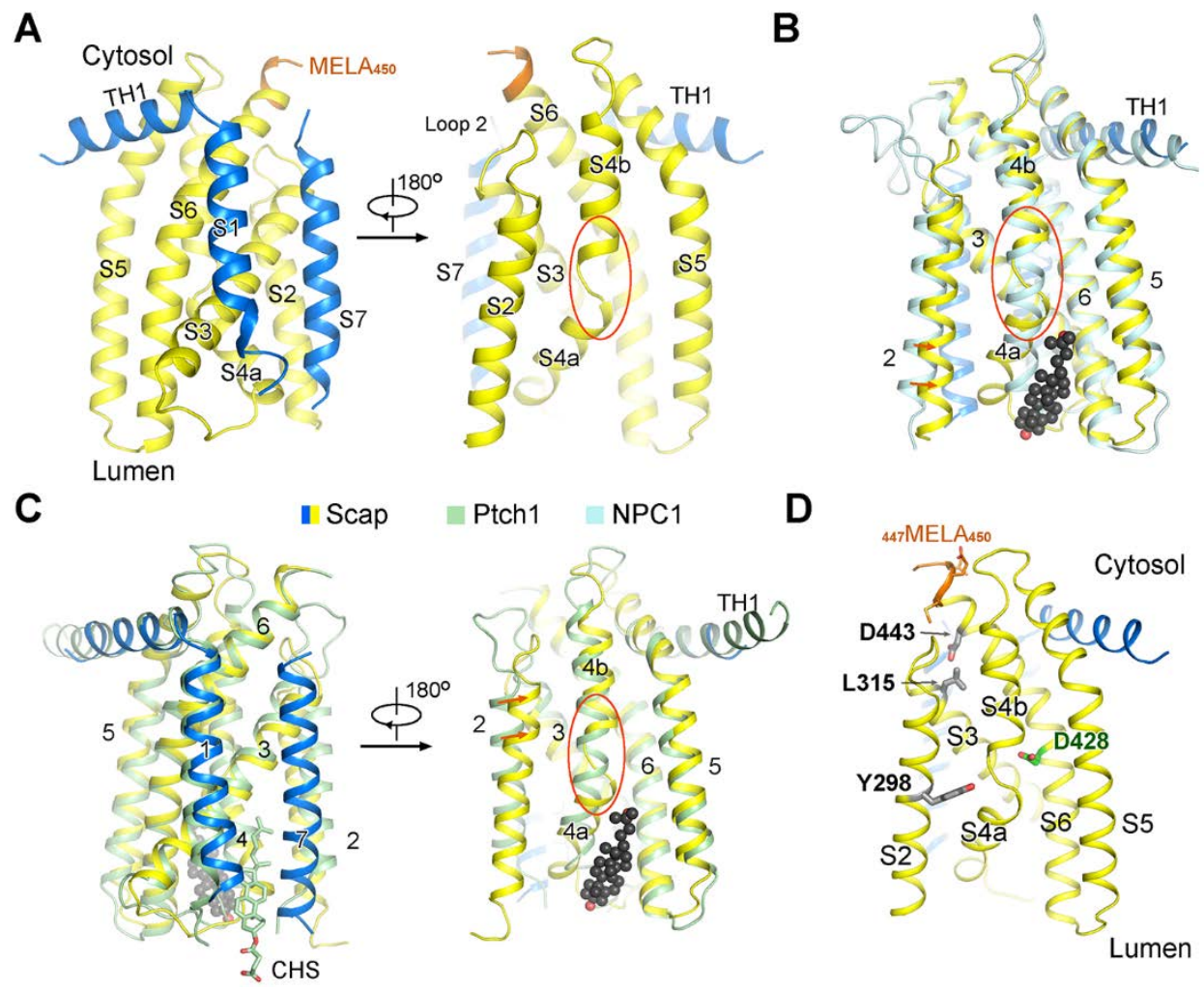

Fig. 2. The S4 segment of Scap is broken in the middle. (A) Structure of the resolved TM domain of Scap. The last four visible residues in S6, ${ }_{447} \mathrm{MELA}_{450}$ from the MELADL motif, are colored orange. The unwound segment in the middle of S4 in Scap is highlighted with an orange circle. Please refer to fig. S4A for detailed annotation of the TM boundaries. (B) SSD comparison between Scap and NPC1 (light cyan). Major structural shifts, indicated by orange arrows, also occur in S2. Of particular note, S4, which is broken in Scap, is an intact helix in Ptch1 and NPC1. (C) SSD comparison between Scap and Patched 1 (Ptch1, light green). It is noted that the potential sterol binding site on Ptch1-SSD, which is occupied by a cholesterol hemisuccinate molecule, is shielded by S7 (or S8) in Scap, while $25 \mathrm{HC}$ binds to the opposite surface of the SSD. The marked shift of S2 is indicated by red arrows. The PDB codes for NPC1 and Ptch1 are 6W5S and $6 \mathrm{DMB}$, respectively. (D) Structural mapping of representative Scap mutations in the TM region. D428A confers Scap the Insig binding ability even in the absence of sterols. Single point mutations Y298C, L315F, and D443N all abolish 25HC-dependent Insig binding. 
A

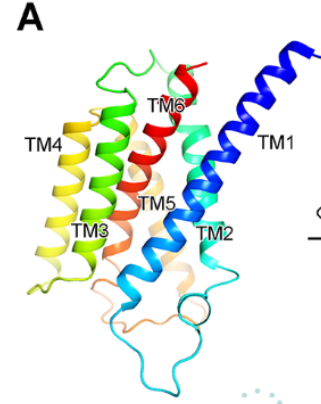

C

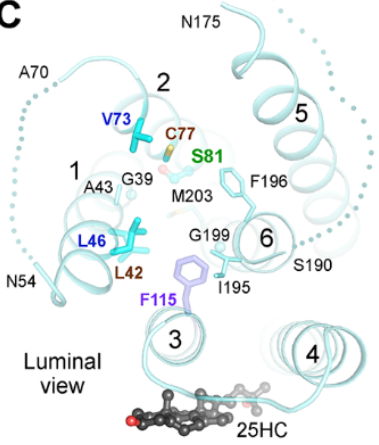

B

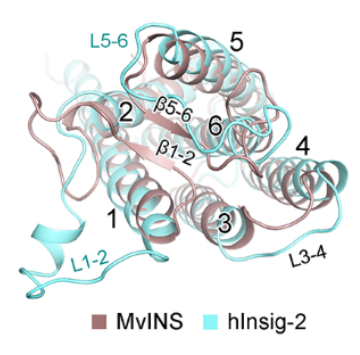

D

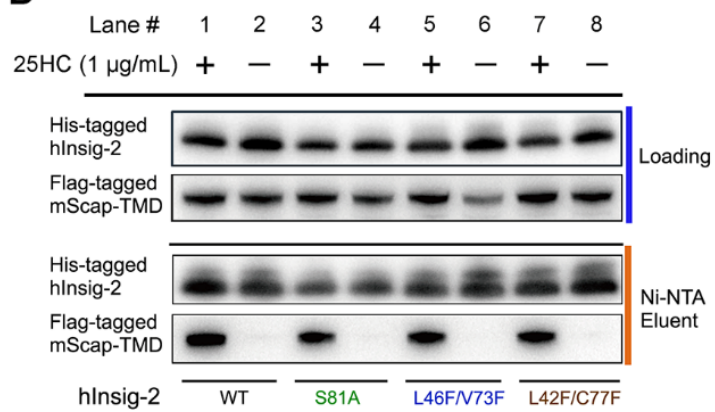

E

\begin{tabular}{|c|c|c|c|c|c|c|c|c|c|c|c|c|}
\hline mScap & \multicolumn{3}{|c|}{ WT } & \multicolumn{3}{c|}{ WT } & \multicolumn{3}{c|}{ WT } & \multicolumn{3}{c|}{ WT } \\
\hline hlnsig-2 & \multicolumn{3}{|c|}{ WT } & \multicolumn{3}{c|}{ S81A } & \multicolumn{3}{c|}{ L46F/N73F } & \multicolumn{3}{c|}{ L42F/C77F } \\
\hline $25 \mathrm{HC}(\mu \mathrm{g} / \mathrm{mL})$ & 0 & 0.1 & 1 & 0 & 0.1 & 1 & 0 & 0.1 & 1 & 0 & 0.1 & 1 \\
\hline Lane & 1 & 2 & 3 & 4 & 5 & 6 & 7 & 8 & 9 & 10 & 11 & 12 \\
\hline
\end{tabular}
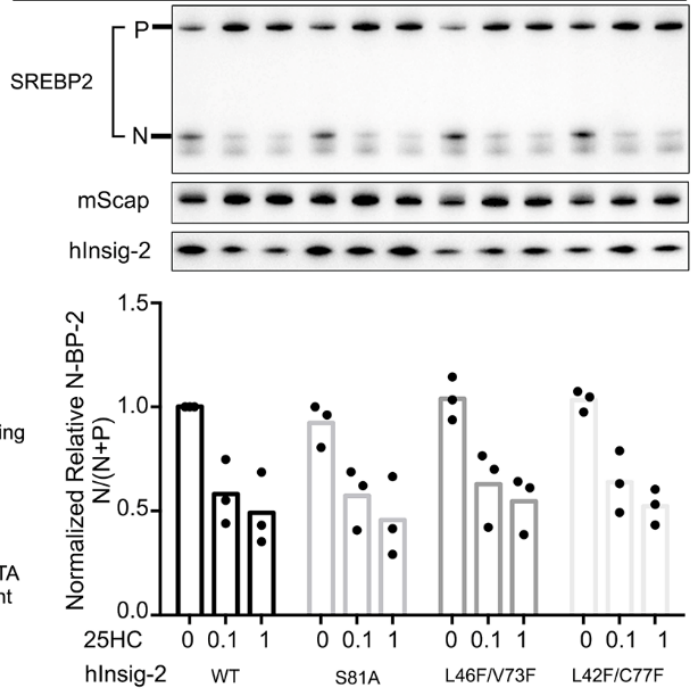

Fig. 3. Structure of human Insig-2. (A) Overall structure of Insig-2. Two opposing side views of Insig-2, rainbow-colored from blue to red, are shown. Please refer to fig. S4B for details of TM boundaries. (B) Structural comparison of human Insig-2 and MvINS, a mycobacterial homolog of Insigs (27). The two structures, shown in a luminal view, can be superimposed with the root-mean-square deviation of $3.3 \AA$ over $141 \mathrm{C} \alpha$ atoms. Note that the TM1-TM2 linker and TM5-TM6 linker in MvINS form antiparallel $\beta$ strands, while the corresponding sequences, which are longer in mammalian Insigs and modeled in panels A and B to indicate the TM connections, were barely resolved in the EM map (fig. S3B). MvINS: Insig from Mycobacterium vanbaalenii (PDB code: 4XU4). (C) A hydrophobic pocket enclosed by TMs 1/2/3/6. A luminal view of Insig-2 is shown. The unresolved TM1-2 and TM5-6 linkers are indicated by dashed lines. (D) Removal of a polar group close to the pocket or blockage of the Insig-2 central pocket with aromatic residues does not interfere with $25 \mathrm{HC}$-dependent Scap binding. The pull-down experiments were performed as reported previously (27) and described in detail in the Materials and methods. Loading and eluted samples are indicated by blue and orange lines on the right of the Western blots. The pull-down results in all figures are representatives of at least three repeated experiments. (E) SREBP cleavage suppression assay for Insig-2 variants containing single or double point mutations S81A, L46F/V73F, and L42F/C77F. The assays were performed using SRD-13A cells that were transfected with $\mathrm{pCMV}-3 \times$ Myc-mScap, indicated pCMV-His-hlnsig-2 variants, and WT pCMV-3×FlaghSREBP2. Top: A representative set of Western blots. "BP2": human SREBP2. "P": the SREBP precursor: "N": the cleaved NTD of SREBP. Bottom: Semi-quantification of the band densities by Image J. The mean ratio of the cleaved form to total SREBP2 $(\mathrm{N} /(\mathrm{N}+\mathrm{P}))$ for WT proteins in the absence of $25 \mathrm{HC}$ in three experiments was set as 1, against which the ratio of other groups was normalized. Please refer to fig. S5 for the original Western blot images. Experimental details are presented in the Materials and methods. 


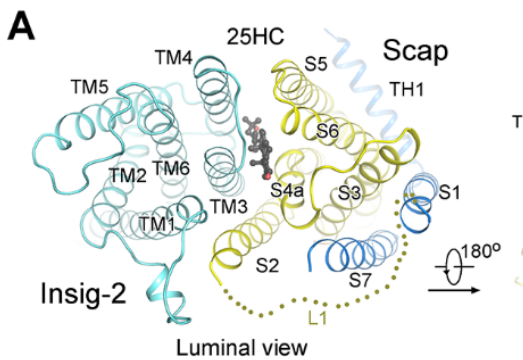

C

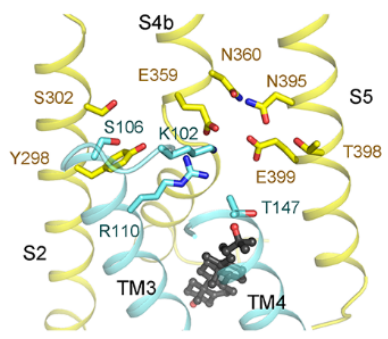

D

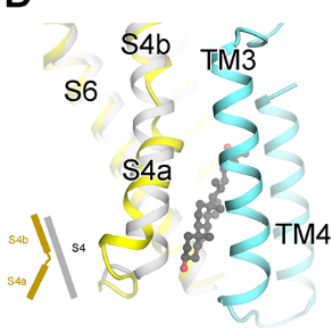

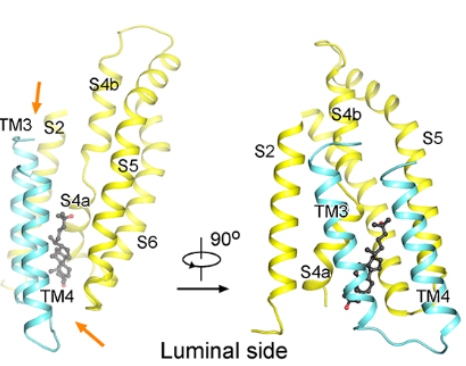

B

E
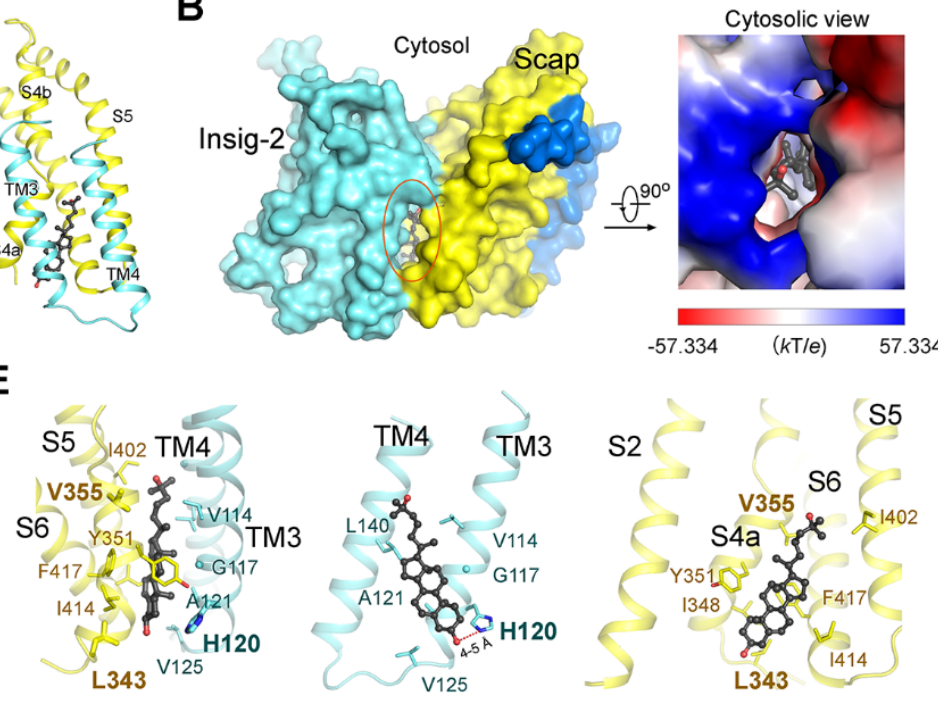

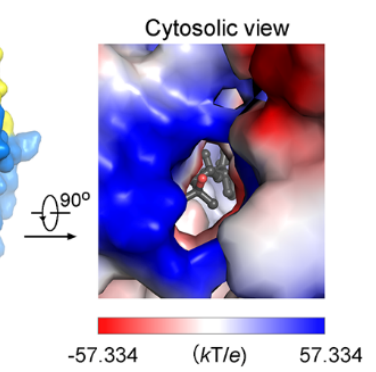

$\mathbf{F}$

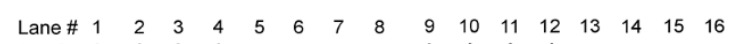
$25 \mathrm{HC}++++---2++++-t_{-}$ His-tagged
hlnsig-2

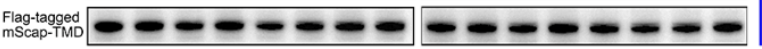
His-tagged
hlnsig-2 Flag-tagged
mScap-TMD $-\ldots-\cdots$

mScap WT WT 1343A V355A WT WT L343A V355A D423A D423A L343ANV355A D423A D423A L343A/V355N hlnsig-2 WT H120A WT WT WT H120A WT WT WT H120A WT WT WT H120A WT WT

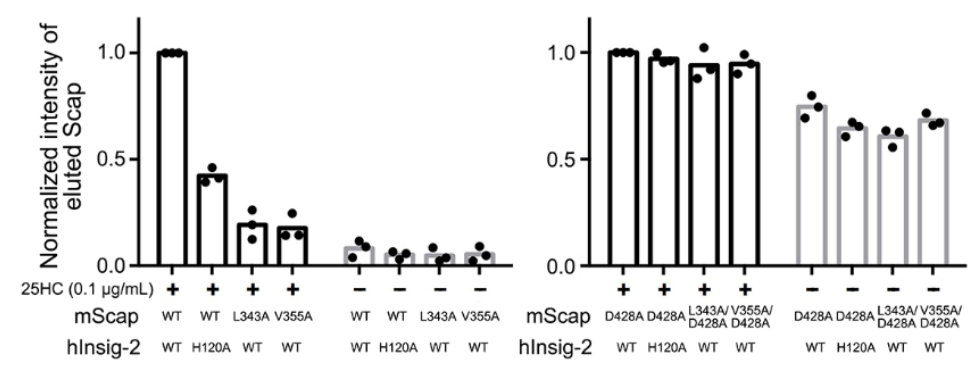

Fig. 4. 25HC mediates the interaction between Scap and Insig-2. (A) The interface between Scap and Insig2. The direct interaction of the two proteins is mainly mediated by Scap-S2/4 and Insig-TM1/3. Please refer to fig. S6 for a detailed illustration of the interaction between Scap and Insig-2 residues. (B) Limited contact between Scap and Insig-2 within the membrane results in a cavity that exposes the $25 \mathrm{OH}$-group of $25 \mathrm{HC}$ to the cytosol. Left: A fenestration on one side of the complex due to the limited contact between Scap-S5 and Insig-TM4. Right: The $25-\mathrm{OH}$ end of the $25 \mathrm{HC}$ tail is exposed to the aqueous environment of the cytosol. Surface electrostatic potential of the complex was calculated in PyMol. (C) The end of the iso-octanol tail is in the vicinity of a cluster of polar and charged residues. The hydrophilic environment provides a potential interpretation for the preference of $25 \mathrm{HC}$ over cholesterol in facilitating the interaction between Scap and Insig-2. (D) Unwinding and bending of Scap-S4 results in a cavity on the Scap/Insig-2 interface for ligand accommodation. The SSD of NPC1 (PDB code: 6W5S, colored dark grey) is superimposed to Scap. A straight Scap-S4 in the complex would clash with 25HC. A cartoon that illustrates the bending of S4 is shown on the bottom left. (E) Coordination of $25 \mathrm{HC}$ by Scap and Insig-2. Left: The backbone of $25 \mathrm{HC}$ is surrounded by hydrophobic residues from both proteins. Middle: Coordination of $25 \mathrm{HC}$ by Insig-2. Potential water-mediated polar interaction between the $3 \beta-\mathrm{OH}$ group of $25 \mathrm{HC}$ and His120 in Insig-2 is indicated by a red, dashed line, although water molecules cannot be resolved at this resolution. Right: Scap mainly engages hydrophobic residues to coordinate $25 \mathrm{HC}$. (F) Functional validation of the structure-revealed $25 \mathrm{HC}$ binding site. The GOF variant Scap(D428A) can form complex with Insig-2 in the absence of sterols (43). Ala substitution of 25HCcoordinating residues in Scap (Leu343 and Val355) and Insig-2 (His120) significantly compromised 25HCdependent complex formation (Lanes 1-4), but only had minor impact on the direct interaction between Scap and Insig-2 in the absence of 25HC in the context of Scap(D428A) (Lanes 9-13). Left: Representative Western blots for the pull down assays. Right: A semiquantitative analysis of the pull-down assays independently repeated for three times. In the two panels, the band densities of eluted Scap (bottom bot), WT in Lane \#1 and D428A in Lane \#9, were respectively set as 1 and served as the normalization reference for the other bands in the same gel. Please refer to the Materials and methods for experimental details, fig. S7 for full blots, and fig. S8 for biochemical characterization of the mutants. 

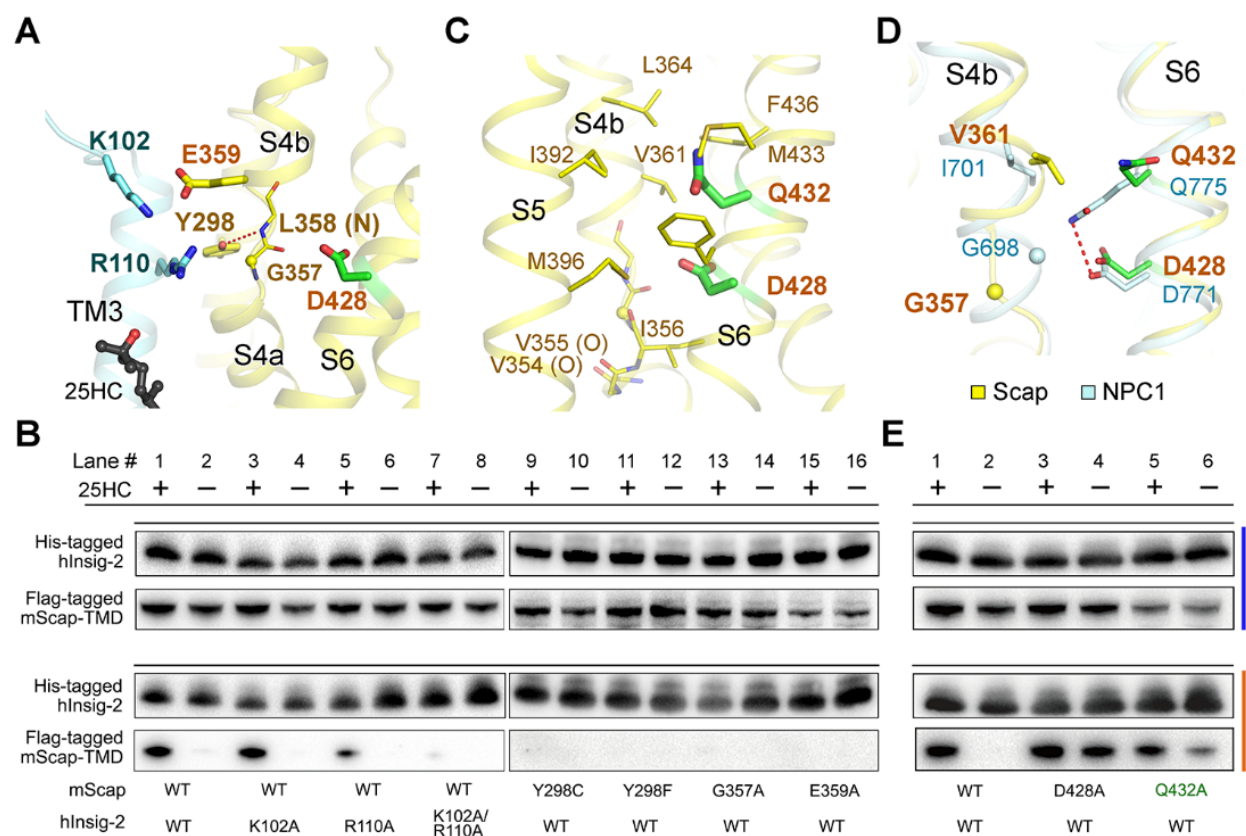

E

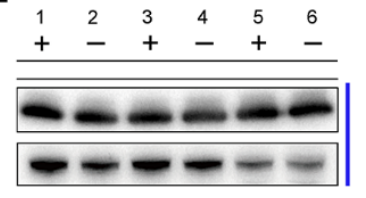

$\mathbf{F}$

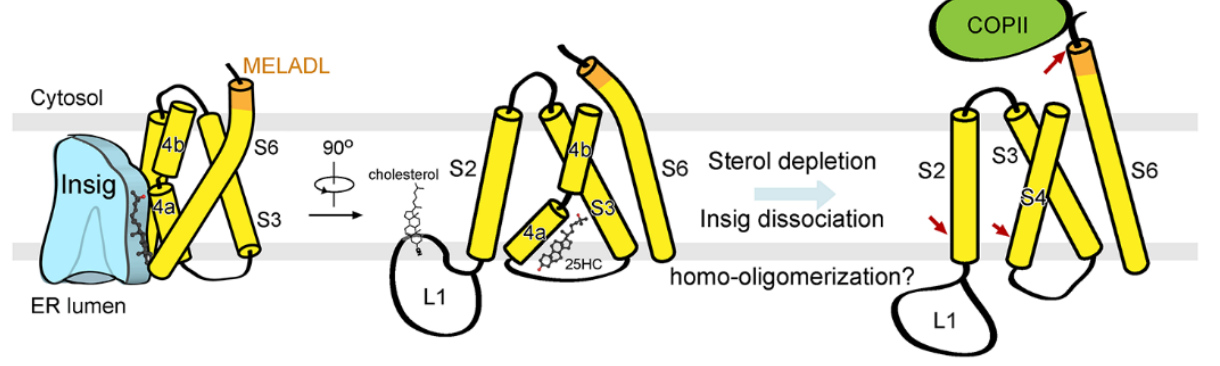

Fig. 5. Unwinding of Scap-S4 is crucial for Insig binding. (A) Unwinding and bending of S4 is stabilized by the bound $25 \mathrm{HC}$ and nearby residues from both Scap and Insig. Lys102 and Arg110 of Insig-2 may simultaneously interact with Scap-Glu359. Unwinding of S4 is important to accommodate Arg110 in Insig-2 and Tyr298 in Scap, which in turn stabilize the mid-point discontinuity of S4. The polar interaction between Tyr298 and the exposed amide of Leu358 in Scap is indicated by a red, dashed line. (B) Functional validation of the key interacting residues shown in panel A. The Scap mutation G357A was designed to prevent helix unwinding. (C) A potential mechanistic interpretation for the GOF of Scap(D428A). Asp428 and Gln432 are both placed unfavorably in a hydrophobic environment within the SSD. Ala substitution of these two polar residues may lower the energy for S4 unwinding, allowing for complex formation with Insig even in the absence of $25 \mathrm{HC}$. In a WT protein, the penalty has to be compensated by sterol binding. (D) Asp428 and Gln432 may prefer S4 in a straight conformation. Asp771 and Gln775, the NPC1 (light cyan) residues corresponding to Asp428 and GIn432, respectively, interact. Scap-Gln432 in a similar conformation as NPC1-GIn775 would clash with Val361 on S4b in the S4-unwound structure, whereas a straight S4 would place Gly357 to face these two residues, leaving enough space for them to interact. (E) Q432A of Scap is also a GOF mutation. Scap (Q432A), to a lesser extent than Scap(D428A), interacts with Insig-2 in the absence of $25 \mathrm{HC}$. Please refer to fig. S8 for biochemical characterization of the mutants and fig. S10 for full blots. (F) A simplified model for 25HC-regulated interaction between Scap and Insig. Left: A cartoon of the structural finding reported in this paper. Middle: In the presence of $25 \mathrm{HC}$ and Insig, S4 of Scap unwinds in the middle, and the luminal Loopl is anchored by cholesterol to the membrane (41). The MELADL motif (orange) is sequestered from being recognized by COPII. Right: In the absence of the sterols, S4 may exist as an intact helix as in other SSD-containing proteins. Conformational changes of multiple SSD segments expose the MELADL motif for COPII recognition. 